\title{
Second class currents and $T$ violation in quasielastic neutrino and antineutrino scattering from nucleons
}

\author{
A. Fatima, M. Sajjad Athar, and S. K. Singh \\ Department of Physics, Aligarh Muslim University, Aligarh-202002, India
}

(Received 22 June 2018; published 24 August 2018)

\begin{abstract}
The effect of the second class currents with and without time reversal invariance has been studied in the quasielastic production of nucleons and hyperons induced by neutrinos and antineutrinos from the nucleons. The numerical results are presented for the total scattering cross section $(\sigma)$ as well as for the longitudinal, perpendicular, and transverse components of the polarization of the final baryons $\left(p, n, \Lambda, \Sigma^{-}, \Sigma^{0}\right)$ and muon produced in the quasielastic (anti)neutrino-nucleon scattering induced by the weak charged current. In the case of the production of the $\Lambda$ hyperon, which is the most suitable candidate for making the polarization measurements, we have also calculated the $Q^{2}$ dependence of the polarization observables and the differential scattering cross section $\left(d \sigma / d Q^{2}\right)$. The measurement of the polarization observables and their $Q^{2}$ dependence provides an independent way to determine the nucleon-hyperon transition form factors at high $Q^{2}$ which can provide tests of the symmetries of the weak hadronic currents like $\mathrm{G}$ invariance, $\mathrm{T}$ invariance, and $\mathrm{SU}(3)$ symmetry.
\end{abstract}

DOI: 10.1103/PhysRevD.98.033005

\section{INTRODUCTION}

The study of the second class currents (SCC) [1] in the weak interaction phenomenology has been pursued for a long time in the low energy processes of $\beta$ decays and muon capture from the nucleons and nuclei [2-7] but there are very few experiments in the high energy region of elastic and quasielastic (anti)neutrino scattering where such studies have been made [8-10]. In the strangeness sector, there are some studies done on the semileptonic decays of polarized hyperons where the polarization and/or the angular correlations of the final leptons are analyzed to see the effect of the SCC [11-18]. Theoretically, however, there are many attempts in the past where the strength of the SCC couplings have been calculated in various models proposed for the nucleon structure [19-30]. Most of these studies have been done assuming the validity of the time reversal invariance (TRI) which requires the strength of the SCC couplings to be real as in the case of the first class currents [31-35]. However, if the strength of the SCC is assumed to be described by a quantity which is complex or purely imaginary then it implies the violation of TRI leading to the time reversal violating (TRV) observables [32-46]. In the past, there have been some theoretical

\footnotetext{
*sajathar@gmail.com
}

Published by the American Physical Society under the terms of the Creative Commons Attribution 4.0 International license. Further distribution of this work must maintain attribution to the author(s) and the published article's title, journal citation, and DOI. Funded by SCOAP. calculations to study the effect of the SCC with or without assuming TRI in the (anti)neutrino scattering from the nucleons [36-48], and suggestions to experimentally study these TRV observables in the $\Delta S=0$ sector [47-49] in the bubble chamber experiments at CERN and FNAL. However, no attempts were made due to the difficulties associated with the measurements of these observables in performing such experiments. While there are few experiments done to study SCC in (anti)neutrino scattering in the $\Delta S=0$ sector assuming TRI [8-10], there is one experiment in the $\Delta S=1$ sector done at CERN [50] where the SCC have been studied in the (anti)neutrino scattering with, as well as without, assuming TRI.

In modern times, very intense neutrino and antineutrino beams are being used to do (anti)neutrino experiments at various laboratories around the world with advanced detector technology [51-59]. These experiments are making measurements of various observables, i.e., the differential cross sections and the angular as well as the energy distributions of the leptons and baryons produced in the final state. The results are interpreted using the standard model (SM) of the electroweak interactions assuming the absence of the second class currents and the validity of TRI. It seems to be the appropriate time to examine the feasibility of making measurements of those observables which enable us to study the effect of the SCC with and without the assumption of TRI. The present work is done with the aim of identifying the suitable observables in the quasielastic scattering of neutrinos and antineutrinos from the nucleons where the effect of the SCC with or without 
TRI make important contributions and can be studied experimentally in the near future.

Generally, the effect of the SCC is small in the differential cross sections and the angular as well as the energy distributions of the final leptons and baryons produced in the $\nu_{e}\left(\bar{\nu}_{e}\right)$ and $\nu_{\mu}\left(\bar{\nu}_{\mu}\right)$ scattering off the nucleons. This is because the leading-order contributions of the second class currents to the differential cross sections, which arise due to the interference of the first and second class currents, are linear in their coupling strengths, are either proportional to $m_{l}^{2} / M^{2}$ ( $m_{l}$ being the lepton mass and $\mathrm{M}$ is the nucleon mass) and/or $\Delta^{2} / M^{2}$ ( $\Delta$ being the mass difference between the initial and final baryons) and are, therefore, almost negligible in the reactions induced by $\nu_{e}\left(\bar{\nu}_{e}\right)$ and $\nu_{\mu}\left(\bar{\nu}_{\mu}\right)$ in the $\Delta S=0$ sector. The contributions which are independent of $m_{l}^{2} / M^{2}$ and $\Delta^{2} / M^{2}$ depend quadratically on the strength of the SCC and are small unless the SCC couplings are enormously large [60,61]. Moreover, their dependence on the momentum transfer is $O\left(Q^{2} / M^{2}\right)$ or higher and their contribution is quantitatively significant only at very high $Q^{2}$, where the differential cross sections become very small making it difficult to isolate the contribution of the SCC. The situation is different in the case of the polarization observables of the leptons and baryons produced in the final state where the leading-order contributions linear in the strength of the SCC couplings also contain terms which are independent of $m_{l}^{2} / M^{2}$ and $\Delta^{2} / M^{2}$. Moreover, in the $\Delta S=1$ sector where hyperons are produced, the contributions of $\Delta^{2} / M^{2}$ terms are not negligible and could be important in favorable kinematics. In view of this, various calculations on the polarization observables of muons and baryons produced in the final state of the (anti)neutrino and electron induced weak processes have been done by many authors [32-48,62-68]. Alternatively, in the case of the reactions where $\tau$ leptons are produced, the terms proportional to $m_{l}^{2} / M^{2}$ become quite significant even in the absence of the SCC. Motivated by these considerations some calculations of the polarization observables of the $\tau$ leptons have been done recently in the weak processes induced by $\tau$ neutrinos [68-73]. The study of the $Q^{2}$ dependence of the polarization observables of the electrons and protons in the scattering of the polarized electrons on protons has played an important role in determining the electromagnetic form factors of the nucleon [74,75]. Similar studies of the polarization observables in the weak sector of the $\Delta S=0$ and $\Delta S=1$ reactions where polarization of the muons and baryons and their $Q^{2}$ dependence made in the quasielastic and inelastic (anti)neutrino scattering from nucleons can play crucial role in determining the weak transition form factors of nucleons.

In the case of the weak $\Delta S=1$ reactions corresponding to the hyperon production, i.e.,

$$
\bar{\nu}_{\mu}+p \rightarrow \mu^{+}+\Lambda\left(\Sigma^{0}\right)
$$

$$
\bar{\nu}_{\mu}+n \rightarrow \mu^{+}+\Sigma^{-},
$$

the final hyperons decay predominantly into pions through two-body weak decays like $\Lambda\left(\Sigma^{0}\right) \rightarrow p \pi^{-}$or $n \pi^{0}$, and $\Sigma^{-} \rightarrow n \pi^{-}$and the asymmetry in the angular distribution of the pions with respect to a given direction (which can be chosen to be the direction of the momentum of the hyperon, perpendicular to it or transverse to the reaction plane) determines the polarization component of the hyperon in that direction. In the case of $\Sigma^{0}$, which promptly decays to $\Lambda$ through the electromagnetic mode of decay, $\Sigma^{0} \rightarrow \Lambda \gamma$ before the weak decay of $\Sigma^{0}$ into pions $p \pi^{-}$or $n \pi^{0}$, the pions coming from such $\Lambda$ decays can be experimentally identified to come from $\Sigma^{0}[50]$ and the asymmetries can be determined. This makes the polarization measurements on the hyperon production relatively easier than the polarization of the nucleon produced in $\Delta S=0$ reactions like

$$
\begin{gathered}
\nu_{\mu}+n \rightarrow \mu^{-}+p \\
\bar{\nu}_{\mu}+p \rightarrow \mu^{+}+n,
\end{gathered}
$$

where double scattering experiments with the final nucleons are required. Such double scattering experiments with the nucleons produced in the neutrino and antineutrino scattering were proposed in the days of bubble chamber experiments at CERN and FNAL by Block and his collaborators $[47,48]$ but were not done at that time. These experiments are difficult and feasibility of doing them in future could be explored with the liquid argon time projection chamber (LArTPC) detectors [51-54].

In view of the above discussion, we have in this work considered the quasielastic production of the nucleons and hyperons induced by (anti)neutrinos and studied the effect of the SCC with and without TRI on the differential cross sections and the polarization observables of the leptons and baryons in the final state. Similar studies have been recently done by us in the weak quasielastic scattering induced by electrons on protons $[65,66]$.

In Sec. II, we describe the structure of the matrix elements for the reactions given in Eqs. (1)-(4) using the SM with three flavors of quarks and discuss the form

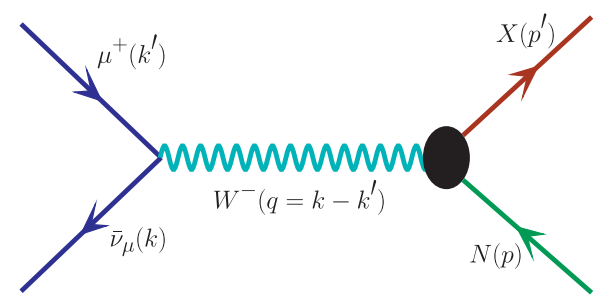

FIG. 1. Feynman diagram for the process $\bar{\nu}_{\mu}(k)+N(p) \rightarrow$ $\mu^{+}\left(k^{\prime}\right)+X\left(p^{\prime}\right)$, where $N(=p, n)$ and $X\left(=n, \Lambda, \Sigma^{0}, \Sigma^{-}\right)$represents the initial nucleon and the final baryon, respectively. The quantities in the bracket represent four momenta of the corresponding particles. 
factors corresponding to the first and the SCC using the implications of SU(3) symmetry. The hypothesis of the conserved vector current (CVC) for the vector currents and the partially conserved axial vector current (PCAC) for the axial vector currents are assumed. In Sec. III, we present the formalism for calculating the differential cross sections and the polarization observables of the final muons and baryons in the presence of the SCC with and without TRI. In Sec. IV, we present and discuss our results while the summary and conclusions are given in Sec. V.

\section{MATRIX ELEMENT AND TRANSITION FORM FACTORS}

\section{A. Matrix element}

The transition matrix element for the processes

$$
\begin{gathered}
\nu_{\mu}(k)+n(p) \rightarrow \mu^{-}\left(k^{\prime}\right)+p\left(p^{\prime}\right), \\
\bar{\nu}_{\mu}(k)+p(p) \rightarrow \mu^{+}\left(k^{\prime}\right)+n\left(p^{\prime}\right), \\
\bar{\nu}_{\mu}(k)+p(p) \rightarrow \mu^{+}\left(k^{\prime}\right)+\Lambda\left(p^{\prime}\right), \\
\bar{\nu}_{\mu}(k)+p(p) \rightarrow \mu^{+}\left(k^{\prime}\right)+\Sigma^{0}\left(p^{\prime}\right), \\
\bar{\nu}_{\mu}(k)+n(p) \rightarrow \mu^{+}\left(k^{\prime}\right)+\Sigma^{-}\left(p^{\prime}\right),
\end{gathered}
$$

shown by the Feynman diagram in Fig. 1, is written as

$$
\mathcal{M}=\frac{G_{F}}{\sqrt{2}} a l^{\mu} J_{\mu}
$$

where the quantities in the brackets of Eqs. (5)-(9) represent the four momenta of the particles, $G_{F}$ is the Fermi coupling constant, $a=\cos \theta_{c}$ for $\Delta S=0$ processes, i.e., Eq. (5) and (6), $a=\sin \theta_{c}$ for $\Delta S=1$ processes, i.e., Eqs. (7)-(9), and $\theta_{c}\left(=13.1^{\circ}\right)$ is the Cabibbo mixing angle. The leptonic current $l^{\mu}$ is given by

$$
l^{\mu}=\bar{u}\left(k^{\prime}\right) \gamma^{\mu}\left(1 \pm \gamma_{5}\right) u(k)
$$

where $(+)$ - sign is for (anti)neutrino. The hadronic current $J_{\mu}$ is expressed as

$$
J_{\mu}=\bar{u}\left(p^{\prime}\right) \Gamma_{\mu} u(p)
$$

with

$$
\Gamma_{\mu}=V_{\mu}-A_{\mu} .
$$

The vector $\left(V_{\mu}\right)$ and the axial vector $\left(A_{\mu}\right)$ currents are given by $[64,65]$ :

$$
\left\langle X\left(p^{\prime}\right)\left|V_{\mu}\right| N(p)\right\rangle=\bar{u}\left(p^{\prime}\right)\left[\gamma_{\mu} f_{1}^{N X}\left(Q^{2}\right)+i \sigma_{\mu \nu} \frac{q^{\nu}}{M+M^{\prime}} f_{2}^{N X}\left(Q^{2}\right)+\frac{2 q_{\mu}}{M+M^{\prime}} f_{3}^{N X}\left(Q^{2}\right)\right] u(p)
$$

and

$$
\left\langle X\left(p^{\prime}\right)\left|A_{\mu}\right| N(p)\right\rangle=\bar{u}\left(p^{\prime}\right)\left[\gamma_{\mu} \gamma_{5} g_{1}^{N X}\left(Q^{2}\right)+i \sigma_{\mu \nu} \frac{q^{\nu}}{M+M^{\prime}} \gamma_{5} g_{2}^{N X}\left(Q^{2}\right)+\frac{2 q_{\mu}}{M+M^{\prime}} g_{3}^{N X}\left(Q^{2}\right) \gamma_{5}\right] u(p),
$$

which is also expressed as

$$
\left\langle X\left(p^{\prime}\right)\left|A_{\mu}\right| N(p)\right\rangle=\bar{u}\left(p^{\prime}\right)\left[\gamma_{\mu} \gamma_{5} g_{1}^{N X}\left(Q^{2}\right)+\left(\frac{\Delta}{M+M^{\prime}} \gamma_{\mu} \gamma_{5}-\frac{p_{\mu}+p_{\mu}^{\prime}}{M+M^{\prime}} \gamma_{5}\right) g_{2}^{N X}\left(Q^{2}\right)+\frac{2 q_{\mu}}{M+M^{\prime}} g_{3}^{N X}\left(Q^{2}\right) \gamma_{5}\right] u(p),
$$

where $X$ represents a nucleon $N(=n, p)$ or a hyperon $Y\left(=\Lambda, \Sigma^{0}\right.$ and $\left.\Sigma^{-}\right), \Delta=M^{\prime}-M$ with $M$ and $M^{\prime}$ being the masses of the initial and final baryons. $q_{\mu}\left(=k_{\mu}-k_{\mu}^{\prime}=p_{\mu}^{\prime}-p_{\mu}\right)$ is the four momentum transfer with $Q^{2}=-q^{2}, Q^{2}>0 . f_{1}^{N X}\left(Q^{2}\right)$, $f_{2}^{N X}\left(Q^{2}\right)$ and $f_{3}^{N X}\left(Q^{2}\right)$ are the vector, weak magnetic and induced scalar form factors and $g_{1}^{N X}\left(Q^{2}\right), g_{2}^{N X}\left(Q^{2}\right)$ and $g_{3}^{N X}\left(Q^{2}\right)$ are the axial vector, induced tensor (or weak electric), and induced pseudoscalar form factors, respectively. Note the difference in the coefficients containing the mass terms appearing with the form factors $f_{3}^{N X}\left(Q^{2}\right)$ and $g_{3}^{N X}\left(Q^{2}\right)$ from those defined in Ref. [65]. We also define here the matrix elements of the electromagnetic current for protons and neutrons in terms of the electromagnetic form factors of the nucleons as

$$
\begin{aligned}
\left\langle p\left(p^{\prime}\right)\left|J_{\mu}^{e m}\right| p(p)\right\rangle & =\bar{u}\left(p^{\prime}\right)\left[\gamma_{\mu} f_{1}^{p}\left(Q^{2}\right)+i \sigma_{\mu \nu} \frac{q^{\nu}}{2 M} f_{2}^{p}\left(Q^{2}\right)\right] u(p), \\
\left\langle n\left(p^{\prime}\right)\left|J_{\mu}^{e m}\right| n(p)\right\rangle & =\bar{u}\left(p^{\prime}\right)\left[\gamma_{\mu} f_{1}^{n}\left(Q^{2}\right)+i \sigma_{\mu \nu} \frac{q^{\nu}}{2 M} f_{2}^{n}\left(Q^{2}\right)\right] u(p),
\end{aligned}
$$

where $f_{1}^{p, n}\left(Q^{2}\right)$ and $f_{2}^{p, n}\left(Q^{2}\right)$ are, respectively, the Dirac and Pauli form factors for the proton and neutron. 


\section{B. Weak transition form factors}

The weak transition form factors $f_{i}^{N X}\left(Q^{2}\right)$ and $g_{i}^{N X}\left(Q^{2}\right)$ for $X=N(n, p)$ or $Y=\left(\Lambda, \Sigma^{0}\right.$ and $\left.\Sigma^{-}\right)$are determined using Cabibbo theory for the electroweak interactions in the SM with three quark flavors. In this model, only two constant couplings, $f_{1}(=1)$ corresponding to the vector current and $g_{1}(=1)$ corresponding to the axial vector current occur in the matrix elements of the weak charged currents taken between $u(d)$ and $d(u)$ quarks which are considered as point particles. In the case of the matrix elements taken between the nucleon and hyperon states which are composites of three quarks bound by the strong interactions and have finite dimensions, the couplings are no longer constant and acquire a $Q^{2}$ dependence, i.e., $f_{1}\left(Q^{2}\right)$ and $g_{1}\left(Q^{2}\right)$ known as the form factors. Moreover, there are additional form factors which appear due to the finite dimensions of the nucleons and hyperons participating in the weak processes and obey the symmetries of the strong interactions responsible for giving the nucleons and hyperons their finite size. Using the general principles of Lorentz covariance, it is well known that the two additional terms $f_{2}\left(Q^{2}\right)$ and $f_{3}\left(Q^{2}\right)$ in the vector sector and the two additional terms $g_{2}\left(Q^{2}\right)$ and $g_{3}\left(Q^{2}\right)$ in the axial vector sector appear in Eq. (14) and (15). Weinberg [1] has introduced a classification scheme of these form factors under G-parity, a symmetry of the strong interaction defined as the product of the charge conjugation (C) and rotation in the isospin space (I-space) by $180^{\circ}$ around $\mathrm{y}$-axis which changes neutron into proton (charge symmetry) and is defined as

$$
G=C e^{i \pi I_{y}}
$$

The vector and axial vector currents of the SM transform under G-parity as

$$
G V_{\mu} G^{-1}=V^{\mu}, \quad G A_{\mu} G^{-1}=-A_{\mu} .
$$

The vector and axial vector currents of the SM with form factors $f_{1}\left(Q^{2}\right)$ and $g_{1}\left(Q^{2}\right)$ transform as in Eq. (20) and are termed as the first class currents. Out of the induced form factors, $f_{2}\left(Q^{2}\right)$ and $g_{3}\left(Q^{2}\right)$ also transform as in Eq. (20) in their respective currents and are classified as the first class currents, while the form factors $f_{3}\left(Q^{2}\right)$ and $g_{2}\left(Q^{2}\right)$ transform with opposite sign in their respective currents and are classified as the SCC. Since the SM has only first class currents, terms with $f_{1}\left(Q^{2}\right)$ and $g_{1}\left(Q^{2}\right)$ form factors and the induced currents comprising of $f_{2}\left(Q^{2}\right)$ and $g_{3}\left(Q^{2}\right)$ would contribute while the SCC form factors should vanish, i.e.,

$$
\begin{aligned}
& f_{3}\left(Q^{2}\right)=0, \\
& g_{2}\left(Q^{2}\right)=0,
\end{aligned}
$$

if G-parity is a good quantum number of strong interactions.

In the $\Delta S=0$ sector, the violation of G-parity due to $(u-d)$ mass difference or the intrinsic charge symmetry violation of the strong interactions is very small, and the form factors $f_{3}\left(Q^{2}\right)$ and $g_{2}\left(Q^{2}\right)$ are expected to be very small. Moreover, in the vector sector, the charged weak vector currents $V_{\mu}$ along with the isovector part of the electromagnetic current $\left(J_{\mu}^{e m}\right)$ is assumed to form an isotriplet. Therefore, the principle of the conservation of the electromagnetic current, applied also to the weak currents, leads to the hypothesis of CVC and predicts $f_{3}\left(Q^{2}\right)=0$. This also relates the weak form factors $f_{1}\left(Q^{2}\right)$ and $f_{2}\left(Q^{2}\right)$ to the isovector electromagnetic form factors of the nucleons. However, in the axial vector sector there is no such constraint on the form factor $g_{2}\left(Q^{2}\right)$ and it could be nonvanishing albeit small. It is because of this reason that most of the experiments in $\Delta S=0$ sector are analyzed for the search of the SCC assuming $f_{3}\left(Q^{2}\right)=0$ with a nonvanishing $g_{2}\left(Q^{2}\right)$ which is found to be small $[2-7,60,61]$.

Theoretically, the values of $g_{2}\left(Q^{2}\right)$ (in the case of $p-n$ transition) calculated in various models of the nucleon structure are found to be very small [19-30]. Experimentally they are also found to be small and consistent with zero with large uncertainties in the measurements. The old experimental results are summarized well by Holstein [2] and Wilkinson [3,4], while there are some new measurements of $g_{2}\left(Q^{2}\right)$ in nuclear $\beta$ decay measurements $[6,7]$.

The general properties of the weak form factors consistent with the constraints due to the symmetry properties of the weak hadronic currents are summarized as [32-34]:

(a) T invariance implies that all the form factors $f_{1-3}\left(Q^{2}\right)$ and $g_{1-3}\left(Q^{2}\right)$ are real.

(b) The assumption that the weak vector currents and its conjugate along with the isovector part of the electromagnetic current form an isotriplet implies that the weak vector form factors $f_{1}\left(Q^{2}\right)$ and $f_{2}\left(Q^{2}\right)$ are related to the isovector electromagnetic form factors of the nucleon, i.e., $f_{1,2}^{n p}\left(Q^{2}\right)=f_{1,2}^{p}\left(Q^{2}\right)-f_{1,2}^{n}\left(Q^{2}\right)$.

(c) The principle of CVC of the weak currents implies that $f_{3}\left(Q^{2}\right)=0$.

(d) The principle of $\mathrm{G}$ invariance implies that $f_{3}\left(Q^{2}\right)=0$ and $g_{2}\left(Q^{2}\right)=0$.

(e) The hypothesis of PCAC relates the form factor $g_{3}\left(Q^{2}\right)$ to the form factor $g_{1}\left(Q^{2}\right)$ through the Goldberger-Treiman (GT) relation.

Therefore, the form factor $f_{3}\left(Q^{2}\right)=0$ by the CVC and $\mathrm{G}$ invariance. The form factor $g_{2}\left(Q^{2}\right)=0$ only in the presence of $\mathrm{G}$ invariance. In the case of $\mathrm{G}$ noninvariance, $g_{2}\left(Q^{2}\right)$ is nonvanishing and if it is real, it preserves TRI whereas if it is purely imaginary or complex, the SCC in the axial vector sector violate the TRI. 
In the case of $\Delta S=1$ processes shown in Eqs. (7)-(9), while (a) above is always true, whereas (b) is replaced by a more general relation between the $\Delta S=1$ vector form factors and the electromagnetic nucleon form factors in the limit of exact SU(3) symmetry which is discussed below. The other properties (c)-(e) apply only in the limit of exact $\mathrm{SU}(3)$ symmetry. However, SU(3) symmetry is known to be only an approximate symmetry due to the large mass difference between $u(d)$ and $s$ quarks and explicit dynamical breaking of $\mathrm{SU}(3)$ symmetry leading to nonzero values of $f_{3}\left(Q^{2}\right)$ and $g_{2}\left(Q^{2}\right)$. While $f_{3}\left(Q^{2}\right)$ is still constrained by the CVC, there is no such constraint for $g_{2}\left(Q^{2}\right)$ and it could be large. Indeed some theoretical calculations find the value of $\left|g_{2}^{p \Lambda} / g_{1}^{p \Lambda}\right|$ to be in the range of 0.2 to 0.5 [23,24], whereas others find a larger value $[12,15,20,21,23,24,27,28]$. Experimentally some earlier analysis of semileptonic hyperon decays (SHD) suggest larger values of $g_{2}^{p \Lambda(\Sigma)}$ and a latest experiment [14] reports $\left|g_{2}^{\Xi \Sigma} / f_{1}^{\Xi \Sigma}\right|=-1.77_{-2.0}^{+2.1} \pm 0.5$ which is consistent with zero within the large systematic uncertainties of the experiment $[11,14]$. It should be noted that most of these analyses are done assuming a real value of $g_{2}\left(Q^{2}\right)$. Moreover, the most extensive analysis of the experimental data on $\Delta S=1$ SHD finds the effect of SU(3) symmetry breaking to be very small provided the physical masses of the nucleons and the hyperons are used in the analysis [11]. In view of this, we calculate the form factors corresponding to the first class currents, i.e., $f_{1}\left(Q^{2}\right), f_{2}\left(Q^{2}\right), g_{1}\left(Q^{2}\right)$, and $g_{3}\left(Q^{2}\right)$ in a SU(3) symmetric analysis using physical masses for the nucleons and hyperons and take $f_{3}\left(Q^{2}\right)=0$. For $g_{2}\left(Q^{2}\right)$, we take a numerical value guided by some calculations done for $g_{2}\left(Q^{2}\right)$ in the quark model $[23,24,28,29]$ and its value used in earlier studies done on the effect of the SCC and TRI in (anti)neutrino scattering [39-41].

\section{SU(3) symmetry and the weak transition form factors}

The weak vector $\left(V_{\mu}\right)$ and the axial vector $\left(A_{\mu}\right)$ currents corresponding to the $\Delta S=0$ and $\Delta S=1$ hadronic currents whose matrix elements are defined between the states $|N\rangle$ and $|X\rangle$ in Eqs. (5)-(9) are assumed to belong to the octet representation of the $\mathrm{SU}(3)$ and are defined as

$$
\begin{aligned}
V_{i}^{\mu} & =\bar{q} F_{i} \gamma^{\mu} q \\
A_{i}^{\mu} & =\bar{q} F_{i} \gamma^{\mu} \gamma^{5} q,
\end{aligned}
$$

where $F_{i}=\frac{\lambda_{i}}{2}(i=1-8)$ are the generators of flavor SU(3) and $\lambda_{i} \mathrm{~s}$ are the well known Gell-Mann matrices. The generators of the $\mathrm{SU}(3)$ group $F_{i}$ obey the following commutation and anticommutation algebra

$$
\begin{aligned}
{\left[F_{i}, F_{j}\right] } & =i f_{i j k} F_{k} \\
\left\{F_{i}, F_{j}\right\} & =\frac{1}{3} \delta_{i j}+d_{i j k} F_{k}, \quad i, j, k=1-8,
\end{aligned}
$$

where $f_{i j k}$ and $d_{i j k}$ are the structure constants, and are antisymmetric and symmetric, respectively, under the interchange of any two indices [76].

The electromagnetic current $\left(J_{e m}^{\mu}\right)$ and the weak vector $\left(V_{ \pm}^{\mu}\right)$ and the axial vector $\left(A_{ \pm}^{\mu}\right)$ charged currents are defined in terms of $V_{i}^{\mu}$ and $A_{i}^{\mu} ; i=1-8$, as

$$
\begin{aligned}
J_{e m}^{\mu} & =V_{3}^{\mu}+\frac{1}{\sqrt{3}} V_{8}^{\mu}, \\
V_{ \pm}^{\mu} & =\left[V_{1}^{\mu} \pm i V_{2}^{\mu}\right] \cos \theta_{c}+\left[V_{4}^{\mu} \pm i V_{5}^{\mu}\right] \sin \theta_{c} \\
A_{ \pm}^{\mu} & =\left[A_{1}^{\mu} \pm i A_{2}^{\mu}\right] \cos \theta_{c}+\left[A_{4}^{\mu} \pm i A_{5}^{\mu}\right] \sin \theta_{c} .
\end{aligned}
$$

In the Cabibbo theory, isovector electromagnetic current $J_{e m}^{\mu}$ along with the weak vector currents $V_{ \pm}^{\mu}$ are assumed to transform as an octet of vector currents under SU(3). Similarly, the axial vector currents are also assumed to transform as an octet under SU(3). The form factors defined in the matrix element of an octet of the vector (axial vector) currents taken between the octets of the initial and the final baryon states as defined in Eqs. (14)-(18) can, therefore, be expressed in terms of the two couplings of the vector (axial vector) currents corresponding to the symmetric and antisymmetric octets according to the decomposition:

$$
8 \times 8=1+8^{S}+8^{A}+10+\overline{10}+27
$$

and the corresponding SU(3) Clebsch-Gordan coefficients. In general, the expression for the matrix element of the transition between the two states of baryons (say $B_{i}$ and $\left.B_{k}\right)$, through the $\mathrm{SU}(3)$ octet $\left(V_{j}\right.$ or $\left.A_{j}\right)$ of currents can be written as [77]:

$$
\begin{aligned}
& \left\langle B_{i}\left|V_{j}\right| B_{k}\right\rangle=i f_{i j k} F^{V}+d_{i j k} D^{V}, \\
& \left\langle B_{i}\left|A_{j}\right| B_{k}\right\rangle=i f_{i j k} F^{A}+d_{i j k} D^{A} .
\end{aligned}
$$

$F^{V}$ and $D^{V}$ are determined from the experimental data on the electromagnetic form factors, and $F^{A}$ and $D^{A}$ are determined from the experimental data on the semileptonic decays of the nucleons and hyperons. Explicitly, the form factors defined in Eqs. (14)-(16) can be expressed as

$$
\begin{array}{ll}
f_{i}\left(Q^{2}\right)=a F_{i}^{V}\left(Q^{2}\right)+b D_{i}^{V}\left(Q^{2}\right) & i=1,2,3 \\
g_{i}\left(Q^{2}\right)=a F_{i}^{A}\left(Q^{2}\right)+b D_{i}^{A}\left(Q^{2}\right) & i=1,2,3
\end{array}
$$

The Clebsch-Gordan coefficients $a$ and $b$ can be calculated for each transition, if we specify the quantum numbers $\left(\left|I, I_{3}, Y\right\rangle\right)$ of the initial and the final state and the current operators $V_{\mu}, A_{\mu}$ and $J_{\mu}^{e m}$ in the octet representation. A straightforward calculation of the various Clebsch-Gordan coefficients in the case of weak $\Delta S=0, \Delta S=1$ hadronic currents and the electromagnetic currents (in the case of vector currents) gives the values of $a$ and $b$ which are 
TABLE I. Values of the coefficients $a$ and $b$ given in Eqs. (29) and (30).

\begin{tabular}{lccr}
\hline \hline Interaction & Transitions & $a$ & $b$ \\
\hline Electromagnetic vector & $p \rightarrow p$ & 1 & $1 / 3$ \\
& $n \rightarrow n$ & 0 & $-2 / 3$ \\
& $n \rightarrow p$ & 1 & 1 \\
Weak vector and axial vector & $p \rightarrow \Lambda$ & $-\sqrt{\frac{3}{2}}$ & $-\frac{1}{\sqrt{6}}$ \\
& $p \rightarrow \Sigma^{0}$ & $-\frac{1}{\sqrt{2}}$ & $\frac{1}{\sqrt{2}}$ \\
& $n \rightarrow \Sigma^{-}$ & -1 & 1 \\
\hline \hline
\end{tabular}

obtained using Eqs. (24), (25), (27) and (28) and are presented in Table I.

\section{Vector form factors}

In the case of the vector currents, the following equations are obtained for the electromagnetic form factors using the values of $a$ and $b$ from Table I:

$$
f_{i}^{p \rightarrow p}\left(Q^{2}\right)=f_{i}^{p}\left(Q^{2}\right)=F_{i}^{V}\left(Q^{2}\right)+\frac{1}{3} D_{i}^{V}\left(Q^{2}\right) \quad i=1,2
$$

$$
f_{i}^{n \rightarrow n}\left(Q^{2}\right)=f_{i}^{n}\left(Q^{2}\right)=-\frac{2}{3} D_{i}^{V}\left(Q^{2}\right) \quad i=1,2
$$

These equations are solved to determine $F_{i}^{V}\left(Q^{2}\right)$ and $D_{i}^{V}\left(Q^{2}\right)$ in terms of the electromagnetic form factors $f_{1,2}^{p}\left(Q^{2}\right)$ and $f_{1,2}^{n}\left(Q^{2}\right)$ for the protons and neutrons. Once the functions $F_{i}^{V}\left(Q^{2}\right)$ and $D_{i}^{V}\left(Q^{2}\right)$ are determined, all the form factors $f_{1,2}^{N X}\left(Q^{2}\right)$ for the transitions under consideration are determined with the help of the coefficients $a$ and $b$ given in Table I. The expressions for the vector form factors in terms of the electromagnetic form factors $f_{1,2}^{p}\left(Q^{2}\right)$ and $f_{1,2}^{n}\left(Q^{2}\right)$ for the various processes given in Eqs. (5)-(9) are given as

$$
\begin{gathered}
f_{1,2}^{n p}\left(Q^{2}\right)=f_{1,2}^{p}\left(Q^{2}\right)-f_{1,2}^{n}\left(Q^{2}\right), \\
f_{1,2}^{p \Lambda}\left(Q^{2}\right)=-\sqrt{\frac{3}{2}} f_{1,2}^{p}\left(Q^{2}\right), \\
f_{1,2}^{n \Sigma^{-}}\left(Q^{2}\right)=-\left[f_{1,2}^{p}\left(Q^{2}\right)+2 f_{1,2}^{n}\left(Q^{2}\right)\right], \\
f_{1,2}^{p \Sigma^{0}}\left(Q^{2}\right)=-\frac{1}{\sqrt{2}}\left[f_{1,2}^{p}\left(Q^{2}\right)+2 f_{1,2}^{n}\left(Q^{2}\right)\right] .
\end{gathered}
$$

The electromagnetic form factors $f_{1,2}^{p}\left(Q^{2}\right)$ and $f_{1,2}^{n}\left(Q^{2}\right)$ are expressed in terms of the Sachs electric and magnetic form factors $G_{E}^{p, n}\left(Q^{2}\right)$ and $G_{M}^{p, n}\left(Q^{2}\right)$ of the nucleons as

$$
\begin{aligned}
& f_{1}^{p, n}\left(Q^{2}\right)=\left(1+\frac{Q^{2}}{4 M^{2}}\right)^{-1}\left[G_{E}^{p, n}\left(Q^{2}\right)+\frac{Q^{2}}{4 M^{2}} G_{M}^{p, n}\left(Q^{2}\right)\right] \\
& f_{2}^{p, n}\left(Q^{2}\right)=\left(1+\frac{Q^{2}}{4 M^{2}}\right)^{-1}\left[G_{M}^{p, n}\left(Q^{2}\right)-G_{E}^{p, n}\left(Q^{2}\right)\right] .
\end{aligned}
$$

For $G_{E}^{p, n}\left(Q^{2}\right)$ and $G_{M}^{p, n}\left(Q^{2}\right)$ various parametrizations are available in the literature and in our numerical calculations, we have used the parametrization given by Bradford et al. [78].

\section{Axial vector form factors}

The axial vector form factors $g_{i}^{N X}\left(Q^{2}\right)(i=1,2,3)$ are expressed in terms of the two functions $F_{i}^{A}\left(Q^{2}\right)$ and $D_{i}^{A}\left(Q^{2}\right)$ corresponding to the asymmetric and symmetric couplings of the two octets. But we express the form factors $g_{i}^{N X}\left(Q^{2}\right)$ in terms of $g_{i}\left(Q^{2}\right)$ and $x_{i}\left(Q^{2}\right)$ which are defined as

$$
\begin{gathered}
g_{i}\left(Q^{2}\right)=F_{i}^{A}\left(Q^{2}\right)+D_{i}^{A}\left(Q^{2}\right)=g_{i}^{n p}\left(Q^{2}\right) \\
x_{i}\left(Q^{2}\right)=\frac{F_{i}^{A}\left(Q^{2}\right)}{F_{i}^{A}\left(Q^{2}\right)+D_{i}^{A}\left(Q^{2}\right)} ; \quad i=1-3
\end{gathered}
$$

and the expressions for the axial vector transition form factors for the various processes given in Eq. (5)-(9) are given as

$$
\begin{gathered}
g_{1,2,3}^{n p}\left(Q^{2}\right)=g_{A, 2,3}^{n p}\left(Q^{2}\right), \\
g_{1,2,3}^{p \Lambda}\left(Q^{2}\right)=-\frac{1}{\sqrt{6}}\left(1+2 x_{1,2,3}\right) g_{A, 2,3}^{n p}\left(Q^{2}\right), \\
g_{1,2,3}^{n \Sigma^{-}}\left(Q^{2}\right)=\left(1-2 x_{1,2,3}\right) g_{A, 2,3}^{n p}\left(Q^{2}\right), \\
g_{1,2,3}^{p \Sigma^{0}}\left(Q^{2}\right)=\frac{1}{\sqrt{2}}\left(1-2 x_{1,2,3}\right) g_{A, 2,3}^{n p}\left(Q^{2}\right) .
\end{gathered}
$$

In the following we describe the explicit forms of the axial vector form factors used for calculating the numerical results.

(a) Axial vector form factor $g_{1}^{N X}\left(Q^{2}\right)$ : We note from Eq. (41), that $g_{1}^{n p}\left(Q^{2}\right)$ is the axial vector form factor for $n \rightarrow p$ transition which is determined experimentally from the quasielastic (anti)neutrino scattering from the nucleons and is parametrized as

$$
g_{1}^{n p}\left(Q^{2}\right)=g_{A}^{n p}\left(Q^{2}\right)=\frac{g_{A}(0)}{\left(1+\frac{Q^{2}}{M_{A}^{2}}\right)^{2}},
$$

with $g_{A}(0)=1.267$ [11] and $M_{A}=1.026 \mathrm{GeV}$ [79]. The parameter $x_{1}\left(Q^{2}\right)$ occurring in Eqs. (42)-(44) for 
$g_{1}^{N Y}\left(Q^{2}\right)\left(Y=\Lambda, \Sigma^{0}, \Sigma^{-}\right)$is determined at low $Q^{2}$ from the analysis of SHD and is found to be $x_{1}\left(Q^{2} \approx 0\right)=0.364$ [11]. There is no experimental information about the $Q^{2}$ dependence of $x_{1}\left(Q^{2}\right)$, therefore, we assume it to be constant, i.e., $x_{1}\left(Q^{2}\right) \approx$ $x_{1}(0)=0.364$ for convenience.

(b) Second class current form factor $g_{2}^{N X}\left(Q^{2}\right)$ : The expression for $g_{2}^{N Y}\left(Q^{2}\right)$ for $Y\left(=\Lambda, \Sigma^{-}, \Sigma^{0}\right)$ are given in Eqs. (42)-(44) in terms of $g_{2}^{n p}\left(Q^{2}\right)$ and $x_{2}\left(Q^{2}\right)$, where $g_{2}^{n p}\left(Q^{2}\right)$ is parametrized as

$$
g_{2}^{n p}\left(Q^{2}\right)=\frac{g_{2}^{n p}(0)}{\left(1+\frac{Q^{2}}{M_{2}^{2}}\right)^{2}},
$$

in analogy with $g_{1}^{n p}\left(Q^{2}\right)=g_{A}\left(Q^{2}\right)$. There is some information on $g_{2}^{n p}\left(Q^{2}\right)$ from neutrino and antineutrino scattering off the nucleons [8-10]. It is shown that the value of $g_{2}^{n p}(0)$ is correlated with the value of $M_{2}$ used in the analysis. There exists theoretical calculations for the $\operatorname{Re} g_{2}^{n p}(0)$ and $\operatorname{Re} g_{2}^{N Y}(0)$ for $Y=\Lambda, \Sigma^{-}, \Sigma^{0}$ using quark models [23,24,28,29]. There are also some older calculations of $\mathrm{T}$ violating effects in weak processes where, phenomenologically, the values of $\operatorname{Im} g_{2}^{N X}\left(Q^{2}\right)$ have been used in a large range of $1<\operatorname{Im} g_{2}^{p n}(0)<10$, like the works of Fearing et al. $\left(\operatorname{Im} g_{2}^{p n}(0)=1,5,10\right)$ [80], Berman and Veltman $\left(\operatorname{Im} g_{2}^{p n}(0)=3.71,6\right)$ [39] and Fujii and Yamaguchi $\left(\operatorname{Im} g_{2}^{p \Lambda}(0)=1.92\right)$ [41]. However, there is no information about $x_{2}\left(Q^{2}\right)$. In view of this we vary the value of $\operatorname{Re} g_{2}^{n p}(0)$ and $\operatorname{Im} g_{2}^{n p}(0)$ in the range of $0-3$ to study the effect of the SCC in (anti)neutrino scattering [3941] and use $M_{2}=M_{A}$. For the $Q^{2}$ dependence of the form factor, i.e., $g_{2}^{N Y}\left(Q^{2}\right)$, we use the $\mathrm{SU}(3)$ symmetric expressions for $g_{2}^{N Y}\left(Q^{2}\right)$ taken to be of dipole form given in Eq. (46) for the various transitions given in Eqs. (42)-(44), in terms of $g_{2}^{n p}\left(Q^{2}\right)$ and $x_{2}\left(Q^{2}\right)$, treating $x_{2}\left(Q^{2}\right)$ to be constant and take $x_{2}=x_{1}$ for simplicity.

(c) The induced pseudoscalar form factor $g_{3}^{N X}\left(Q^{2}\right)$ : We see from Eqs. (42)-(44), that $g_{3}^{N Y}\left(Q^{2}\right)$ is determined in terms of $g_{3}^{n p}\left(Q^{2}\right)$ and $x_{3}\left(Q^{2}\right)$ for $Y=\Lambda, \Sigma^{0}$ and $\Sigma^{-}$. In general, the contribution of $g_{3}^{n p}\left(Q^{2}\right)$ to the (anti)neutrino scattering cross sections is proportional to $m_{l}^{2}$, where $m_{l}$ is the mass of the corresponding charged lepton, and is very small. There is very little experimental information available about $g_{3}^{n p}\left(Q^{2}\right)$ at very small $Q^{2}$ from the muon capture experiments in the nucleons and nuclei [81]. This information is consistent with the prediction of $g_{3}^{n p}\left(Q^{2}\right)$ obtained using the hypothesis of PCAC and GT relation [82] in the $\Delta S=0$ sector which is given as

$$
g_{3}^{n p}\left(Q^{2}\right)=\frac{2 M^{2} g_{A}^{n p}\left(Q^{2}\right)}{m_{\pi}^{2}+Q^{2}}
$$

There is no information about $x_{3}\left(Q^{2}\right)$ from the analysis of the SHD due to the small contribution of $g_{3}^{N Y}\left(Q^{2}\right)$. In the absence of any information about $x_{3}\left(Q^{2}\right)$, we do not use $\mathrm{SU}(3)$ symmetric expressions for $g_{3}^{N Y}\left(Q^{2}\right)$ given in Eqs. (42)-(44). Instead we use the expression for $g_{3}^{N Y}\left(Q^{2}\right)$ using the generalized PCAC and GT relation [82] for the $\Delta S=1$ currents given by Nambu [83], i.e.,

$$
g_{3}^{N Y}\left(Q^{2}\right)=\frac{\left(M+M_{Y}\right)^{2}}{2\left(m_{K}^{2}+Q^{2}\right)} g_{1}^{N Y}\left(Q^{2}\right),
$$

where $m_{K}$ is the mass of kaon and $g_{1}^{N Y}\left(Q^{2}\right)$ is given in Eqs. (42)-(44), for $Y=\Lambda, \Sigma^{-}, \Sigma^{0}$.

\section{CROSS SECTION AND THE POLARIZATION OBSERVABLES}

\section{A. Cross section}

The general expression of the differential cross section for the processes (5)-(9), in the laboratory frame, is written as

$$
\begin{aligned}
d \sigma= & \frac{1}{(2 \pi)^{2}} \frac{1}{4 M E_{\nu}} \delta^{4}\left(k+p-k^{\prime}-p^{\prime}\right) \\
& \times \frac{d^{3} k^{\prime}}{2 E_{k^{\prime}}} \frac{d^{3} p^{\prime}}{2 E_{p^{\prime}}} \sum \sum|\mathcal{M}|^{2},
\end{aligned}
$$

where $E_{\nu}=\left(E_{\bar{\nu}_{\mu}}\right) E_{\nu_{\mu}}$ is the incoming (anti)neutrino energy. The transition matrix element squared is defined as

$$
\bar{\sum} \sum|\mathcal{M}|^{2}=\frac{G_{F}^{2} a^{2}}{2} \mathcal{J}^{\mu \nu} \mathcal{L}_{\mu \nu}
$$

where the hadronic and the leptonic tensors are obtained using Eqs. (11) and (12) as

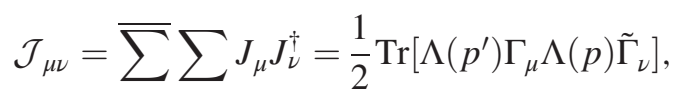

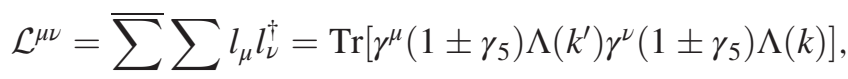

with $\tilde{\Gamma}_{\nu}=\gamma^{0} \Gamma_{\nu}^{\dagger} \gamma^{0}$ and the expression for $\Gamma_{\nu}$ is given in Eq. (13). The spin $\frac{1}{2}$ projection operator $\Lambda(P)$ for momentum $P=k, k^{\prime}, p, p^{\prime}$ corresponding to the initial and the final baryons and the leptons are given by 


$$
\Lambda(P)=\left(\not P+M_{P}\right)
$$

where $M_{P}$ is the mass of the particle with momentum $P$.

Following the above definitions, the differential scattering cross section $d \sigma / d Q^{2}$ for the processes given in Eq. (5)-(9) is written as

$$
\frac{d \sigma}{d Q^{2}}=\frac{G_{F}^{2} a^{2}}{8 \pi M^{2} E_{\nu}^{2}} N\left(Q^{2}\right),
$$

where $N\left(Q^{2}\right)=\mathcal{J}^{\mu \nu} \mathcal{L}_{\mu \nu}$ and the expression of $N\left(Q^{2}\right)$ is given in the Appendix A. The expression of $N\left(Q^{2}\right)$ is consistent with the expression given by Llewellyn Smith [34] in the limit $M^{\prime}=M$ and $g_{2}\left(Q^{2}\right)=0$. In this limit, it is also consistent with Bilenky and Christova [62].

\section{B. Polarization of the final baryon}

Using the covariant density matrix formalism, the polarization 4-vector $\left(\xi^{\tau}\right)$ of the baryon produced in the final state in reactions (5)-(9) is written as [84]:

$$
\xi^{\tau}=\frac{\operatorname{Tr}\left[\gamma^{\tau} \gamma_{5} \rho_{f}\left(p^{\prime}\right)\right]}{\operatorname{Tr}\left[\rho_{f}\left(p^{\prime}\right)\right]},
$$

where the spin density matrix $\rho_{f}\left(p^{\prime}\right)$ corresponding to the final baryon of momentum $p^{\prime}$ is given by

$$
\rho_{f}\left(p^{\prime}\right)=\mathcal{L}^{\alpha \beta} \operatorname{Tr}\left[\Lambda\left(p^{\prime}\right) \Gamma_{\alpha} \Lambda(p) \tilde{\Gamma}_{\beta} \Lambda\left(p^{\prime}\right)\right] .
$$

In the above expression, $\Lambda(p)$ and $\Gamma_{\alpha}$ are given in Eqs. (53) and (13), respectively. Using the following relations:

$$
\Lambda\left(p^{\prime}\right) \gamma^{\tau} \gamma_{5} \Lambda\left(p^{\prime}\right)=2 M^{\prime}\left(g^{\tau \sigma}-\frac{p^{\prime \tau} p^{\prime \sigma}}{M^{\prime 2}}\right) \Lambda\left(p^{\prime}\right) \gamma_{\sigma} \gamma_{5}
$$

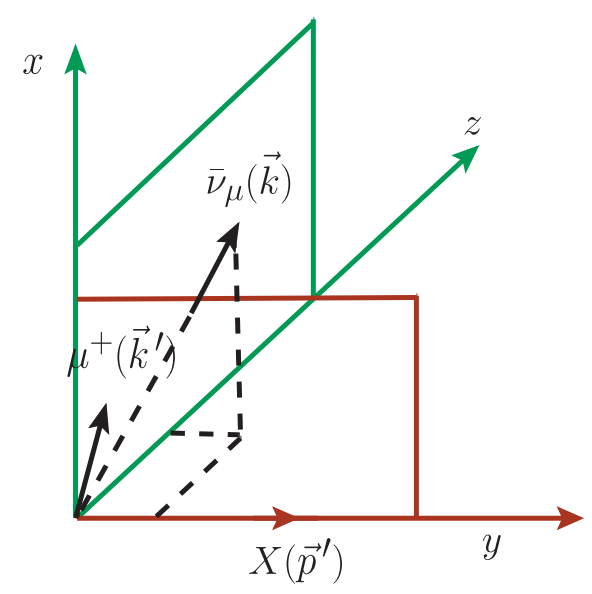

(a)

and

$$
\Lambda\left(p^{\prime}\right) \Lambda\left(p^{\prime}\right)=2 M^{\prime} \Lambda\left(p^{\prime}\right)
$$

$\xi^{\tau}$ defined in Eq. (55) may be rewritten after some algebra as

$\xi^{\tau}=\left(g^{\tau \sigma}-\frac{p^{\prime \tau} p^{\prime \sigma}}{M^{\prime 2}}\right) \frac{\mathcal{L}^{\alpha \beta} \operatorname{Tr}\left[\gamma_{\sigma} \gamma_{5} \Lambda\left(p^{\prime}\right) \Gamma_{\alpha} \Lambda(p) \tilde{\Gamma}_{\beta}\right]}{\mathcal{L}^{\alpha \beta} \operatorname{Tr}\left[\Lambda\left(p^{\prime}\right) \Gamma_{\alpha} \Lambda(p) \tilde{\Gamma}_{\beta}\right]}$.

Note that in Eq. (59), $\xi^{\tau}$ is manifestly orthogonal to $p^{\prime \tau}$, i.e., $p^{\prime} \cdot \xi=0$. Moreover, the denominator is directly related to the differential cross section given in Eq. (54).

With $\mathcal{J}^{\alpha \beta}$ and $\mathcal{L}_{\alpha \beta}$ given in Eqs. (51) and (52), respectively, an expression for $\xi^{\tau}$ is obtained. In the laboratory frame where the initial nucleon is at rest, the polarization vector $\vec{\xi}$ for the final baryon is calculated to be a function of 3 -momenta of incoming antineutrino $(\vec{k})$ and outgoing baryon $\left(\vec{p}^{\prime}\right)$, and is given as

$\vec{\xi}=\left[A^{h}\left(Q^{2}\right) \vec{k}+B^{h}\left(Q^{2}\right) \vec{p}^{\prime}+C^{h}\left(Q^{2}\right) M\left(\vec{k} \times \vec{p}^{\prime}\right)\right]$,

where the expressions of $A^{h}\left(Q^{2}\right), B^{h}\left(Q^{2}\right)$, and $C^{h}\left(Q^{2}\right)$ are given in Appendix A.

We now expand the polarization vector $\vec{\xi}$ along the orthogonal directions, $\hat{e}_{L}^{h}, \hat{e}_{P}^{h}$ and $\hat{e}_{T}^{h}$ in the reaction plane corresponding to the longitudinal, perpendicular, and transverse directions, defined as

$\hat{e}_{L}^{h}=\frac{\vec{p}^{\prime}}{\left|\vec{p}^{\prime}\right|}, \quad \hat{e}_{P}^{h}=\hat{e}_{L}^{h} \times \hat{e}_{T}^{h}, \quad$ where $\quad \hat{e}_{T}^{h}=\frac{\vec{p}^{\prime} \times \vec{k}}{\left|\vec{p}^{\prime} \times \vec{k}\right|}$,

and have depicted in Fig. 2. We then write $\vec{\xi}$ as

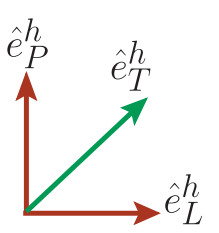

(b)

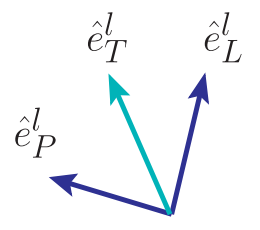

(c)

FIG. 2. (a) Momentum and polarization directions of the final baryon and the lepton. $\hat{e}_{L}^{h, l}, \hat{e}_{P}^{h, l}$ and $\hat{e}_{T}^{h, l}$ represent the orthogonal unit vectors corresponding to the longitudinal, perpendicular, and transverse directions with respect to the momentum of the final baryon in (b) and the final lepton in (c). 


$$
\vec{\xi}=\xi_{L} \hat{e}_{L}^{h}+\xi_{P} \hat{e}_{P}^{h}+\xi_{T} \hat{e}_{T}^{h}
$$

such that the longitudinal, perpendicular, and transverse components of the polarization vector $(\vec{\xi})$ in the laboratory frame are given by

$$
\xi_{L}\left(Q^{2}\right)=\vec{\xi} \cdot \hat{e}_{L}^{h}, \quad \xi_{P}\left(Q^{2}\right)=\vec{\xi} \cdot \hat{e}_{P}^{h}, \quad \xi_{T}\left(Q^{2}\right)=\vec{\xi} \cdot \hat{e}_{T}^{h}
$$

From Eq. (63), the longitudinal $P_{L}^{h}\left(Q^{2}\right)$, perpendicular $P_{P}^{h}\left(Q^{2}\right)$, and transverse $P_{T}^{h}\left(Q^{2}\right)$ components of the polarization vector defined in the rest frame of the initial nucleon are given by

$$
\begin{aligned}
& P_{L}^{h}\left(Q^{2}\right)=\frac{M^{\prime}}{E_{p^{\prime}}} \xi_{L}\left(Q^{2}\right), \quad P_{P}^{h}\left(Q^{2}\right)=\xi_{P}\left(Q^{2}\right), \\
& P_{T}^{h}\left(Q^{2}\right)=\xi_{T}\left(Q^{2}\right),
\end{aligned}
$$

where $\frac{M^{\prime}}{E_{p^{\prime}}}$ is the Lorentz boost factor along $\vec{p}^{\prime}$. With the help of Eqs. (60), (61), (63), and (64), the longitudinal $P_{L}^{h}\left(Q^{2}\right)$, perpendicular $P_{P}^{h}\left(Q^{2}\right)$, and transverse $P_{T}^{h}\left(Q^{2}\right)$ components are calculated to be

$$
\begin{gathered}
P_{L}^{h}\left(Q^{2}\right)=\frac{M^{\prime}}{E_{p^{\prime}}} \frac{A^{h}\left(Q^{2}\right) \vec{k} \cdot \vec{p}^{\prime}+B^{h}\left(Q^{2}\right)\left|\vec{p}^{\prime}\right|^{2}}{N\left(Q^{2}\right)\left|\vec{p}^{\prime}\right|}, \\
P_{P}^{h}\left(Q^{2}\right)=\frac{A^{h}\left(Q^{2}\right)\left[\left(\vec{k} \cdot \vec{p}^{\prime}\right)^{2}-|\vec{k}|^{2}\left|\vec{p}^{\prime}\right|^{2}\right]}{N\left(Q^{2}\right)\left|\vec{p}^{\prime}\right|\left|\vec{p}^{\prime} \times \vec{k}\right|}, \\
P_{T}^{h}\left(Q^{2}\right)=\frac{C^{h}\left(Q^{2}\right) M\left[\left(\vec{k} \cdot \vec{p}^{\prime}\right)^{2}-|\vec{k}|^{2}\left|\vec{p}^{\prime}\right|^{2}\right]}{N\left(Q^{2}\right)\left|\vec{p}^{\prime} \times \vec{k}\right|},
\end{gathered}
$$

which are consistent with the expressions of the components of polarization given by Erriquez et al. [50] except for a change in sign in one of the terms in $P_{P}^{h}\left(Q^{2}\right)$. The above expressions for $P_{L}^{h}\left(Q^{2}\right)$ and $P_{P}^{h}\left(Q^{2}\right)$ are also consistent with the expressions given by Bilenky and Christova [62] in the limit $M^{\prime}=M$ and $g_{2}(0)=g_{3}\left(Q^{2}\right)=0$.

If the T invariance is assumed then all the vector and the axial vector form factors are real and the expression for $C^{h}\left(Q^{2}\right)$ vanishes which implies that the transverse component of the polarization perpendicular to the production plane, $P_{T}^{h}\left(Q^{2}\right)$ vanishes.

\section{Polarization of the final lepton}

Instead of the final baryon polarization if one assumes the final lepton to be polarized, then the polarization 4 -vector $\left(\zeta^{\tau}\right)$ in reaction (5)-(9) is written as

$$
\zeta^{\tau}=\frac{\operatorname{Tr}\left[\gamma^{\tau} \gamma_{5} \rho_{f}\left(k^{\prime}\right)\right]}{\operatorname{Tr}\left[\rho_{f}\left(k^{\prime}\right)\right]},
$$

and the spin density matrix for the final lepton $\rho_{f}\left(k^{\prime}\right)$ is given by

$\rho_{f}\left(k^{\prime}\right)=\mathcal{J}^{\alpha \beta} \operatorname{Tr}\left[\Lambda\left(k^{\prime}\right) \gamma_{\alpha}\left(1 \pm \gamma_{5}\right) \Lambda(k) \tilde{\gamma}_{\beta}\left(1 \pm \tilde{\gamma}_{5}\right) \Lambda\left(k^{\prime}\right)\right]$,

with $\tilde{\gamma}_{\alpha}=\gamma^{0} \gamma_{\alpha}^{\dagger} \gamma^{0}$ and $\tilde{\gamma}_{5}=\gamma^{0} \gamma_{5}^{\dagger} \gamma^{0}$.

Using Eqs. (57) and (58), $\zeta^{\tau}$ defined in Eq. (68) may also be rewritten as

$$
\begin{aligned}
\zeta^{\tau}= & \left(g^{\tau \sigma}-\frac{k^{\prime \tau} k^{\prime \sigma}}{m_{\mu}^{2}}\right) \\
& \times \frac{\mathcal{J}^{\alpha \beta} \operatorname{Tr}\left[\gamma_{\sigma} \gamma_{5} \Lambda\left(k^{\prime}\right) \gamma_{\alpha}\left(1 \pm \gamma_{5}\right) \Lambda(k) \tilde{\gamma}_{\beta}\left(1 \pm \tilde{\gamma}_{5}\right)\right]}{\mathcal{J}^{\alpha \beta} \operatorname{Tr}\left[\Lambda\left(k^{\prime}\right) \gamma_{\alpha}\left(1 \pm \gamma_{5}\right) \Lambda(k) \tilde{\gamma}_{\beta}\left(1 \pm \tilde{\gamma}_{5}\right)\right]},
\end{aligned}
$$

where $m_{\mu}$ is the mass of the muon. In Eq. (70), the denominator is directly related to the differential cross section given in Eq. (54).

With $\mathcal{J}^{\alpha \beta}$ and $\mathcal{L}_{\alpha \beta}$ given in Eqs. (51) and (52), respectively, an expression for $\zeta^{\tau}$ is obtained. In the laboratory frame where the initial nucleon is at rest, the polarization vector $\vec{\zeta}$ is calculated to be a function of 3-momenta of incoming antineutrino $(\vec{k})$ and outgoing lepton $\left(\vec{k}^{\prime}\right)$, and is given as

$$
\vec{\zeta}=\left[A^{l}\left(Q^{2}\right) \vec{k}+B^{l}\left(Q^{2}\right) \vec{k}^{\prime}+C^{l}\left(Q^{2}\right) M\left(\vec{k} \times \vec{k}^{\prime}\right)\right],
$$

where the expressions of $A^{l}\left(Q^{2}\right), B^{l}\left(Q^{2}\right)$, and $C^{l}\left(Q^{2}\right)$ are given in Appendix B.

One may expand the polarization vector $\vec{\zeta}$ along the orthogonal directions, $\hat{e}_{L}^{l}, \hat{e}_{P}^{l}$ and $\hat{e}_{T}^{l}$ in the reaction plane corresponding to the longitudinal, perpendicular and transverse directions, defined as

$\hat{e}_{L}^{l}=\frac{\vec{k}^{\prime}}{\left|\vec{k}^{\prime}\right|}, \quad \hat{e}_{P}^{l}=\hat{e}_{L}^{l} \times \hat{e}_{T}^{l}, \quad$ where $\hat{e}_{T}^{l}=\frac{\vec{k} \times \vec{k}^{\prime}}{\left|\vec{k} \times \vec{k}^{\prime}\right|}$,

and depicted in Fig. 2. We then write $\vec{\zeta}$ as

$$
\vec{\zeta}=\zeta_{L} \hat{e}_{L}^{l}+\zeta_{P} \hat{e}_{P}^{l}+\zeta_{T} \hat{e}_{T}^{l},
$$

such that the longitudinal, perpendicular, and transverse components of the $\vec{\zeta}$ in the laboratory frame are given by $\zeta_{L}\left(Q^{2}\right)=\vec{\zeta} \cdot \hat{e}_{L}^{l}, \quad \zeta_{P}\left(Q^{2}\right)=\vec{\zeta} \cdot \hat{e}_{P}^{l}, \quad \zeta_{T}\left(Q^{2}\right)=\vec{\zeta} \cdot \hat{e}_{T}^{l}$. 
From Eq. (74), the longitudinal $P_{L}^{l}\left(Q^{2}\right)$, perpendicular $P_{P}^{l}\left(Q^{2}\right)$, and transverse $P_{T}^{l}\left(Q^{2}\right)$ components of the polarization vector defined in the rest frame of the initial nucleon are given by

$$
\begin{aligned}
& P_{L}^{l}\left(Q^{2}\right)=\frac{m_{\mu}}{E_{k^{\prime}}} \zeta_{L}\left(Q^{2}\right), \quad P_{P}^{l}\left(Q^{2}\right)=\zeta_{P}\left(Q^{2}\right), \\
& P_{T}^{l}\left(Q^{2}\right)=\zeta_{T}\left(Q^{2}\right),
\end{aligned}
$$

where $\frac{m_{\mu}}{E_{k^{\prime}}}$ is the Lorentz boost factor along $\vec{k}^{\prime}$. Using Eqs. (71), (72), and (74) in Eq. (75), the longitudinal $P_{L}^{l}\left(Q^{2}\right)$, perpendicular $P_{P}^{l}\left(Q^{2}\right)$, and transverse $P_{T}^{l}\left(Q^{2}\right)$ components are calculated to be

$$
\begin{gathered}
P_{L}^{l}\left(Q^{2}\right)=\frac{m_{\mu}}{E_{k^{\prime}}} \frac{A^{l}\left(Q^{2}\right) \vec{k} \cdot \vec{k}^{\prime}+B^{l}\left(Q^{2}\right)\left|\vec{k}^{\prime}\right|^{2}}{N\left(Q^{2}\right)\left|\vec{k}^{\prime}\right|}, \\
P_{P}^{l}\left(Q^{2}\right)=\frac{A^{l}\left(Q^{2}\right)\left[|\vec{k}|^{2}\left|\vec{k}^{\prime}\right|^{2}-\left(\vec{k} \cdot \vec{k}^{\prime}\right)^{2}\right]}{N\left(Q^{2}\right)\left|\vec{k}^{\prime}\right|\left|\vec{k} \times \vec{k}^{\prime}\right|}, \\
P_{T}^{l}\left(Q^{2}\right)=\frac{C^{l}\left(Q^{2}\right) M\left[\left(\vec{k} \cdot \vec{k}^{\prime}\right)^{2}-|\vec{k}|^{2}\left|\vec{k}^{\prime}\right|^{2}\right]}{N\left(Q^{2}\right)\left|\vec{k} \times \vec{k}^{\prime}\right|} .
\end{gathered}
$$

\section{RESULTS AND DISCUSSION}

We have used Eq. (54) to numerically evaluate the differential cross section $d \sigma / d Q^{2}$, Eqs. (65), (66), and (67) to evaluate the longitudinal $P_{L}^{h}\left(Q^{2}\right)$, perpendicular $P_{P}^{h}\left(Q^{2}\right)$, and transverse $P_{T}^{h}\left(Q^{2}\right)$ components of the polarization of the final baryon, and Eqs. (76), (77), and (78) to evaluate the longitudinal $P_{L}^{l}\left(Q^{2}\right)$, perpendicular $P_{P}^{l}\left(Q^{2}\right)$, and transverse $P_{T}^{l}\left(Q^{2}\right)$ components of polarization of the final lepton. The Dirac and Pauli form factors $f_{1,2}^{N}\left(Q^{2}\right)$;
$(N=p, n)$ are expressed in terms of the electric and magnetic Sachs' form factors, for which the parametrization given by Bradford et al. [78] have been used. Using the $\mathrm{SU}(3)$ symmetry, the axial vector form factors, $g_{1}^{N X}\left(Q^{2}\right)$ and $g_{2}^{N X}\left(Q^{2}\right)$ are expressed in terms of $g_{A}^{n p}\left(Q^{2}\right)$ and $g_{2}^{n p}\left(Q^{2}\right)$. For $g_{A}^{n p}\left(Q^{2}\right)$ and $g_{2}^{n p}\left(Q^{2}\right)$, dipole parametrizations have been used as written in Eqs. (45) and (46) with $M_{A}=1.026 \mathrm{GeV}$ and $M_{2}=M_{A}$. For $g_{3}^{n p}\left(Q^{2}\right)$, PCAC along with GT relation has been used and for $g_{3}^{N Y}\left(Q^{2}\right)$, the generalized PCAC along with GT relation given by Nambu [83] has been used to relate them, respectively, with $g_{1}^{n p}\left(Q^{2}\right)$ and $g_{1}^{N Y}\left(Q^{2}\right)$ as shown in Eqs. (47) and (48). To study the dependence of the cross section $\sigma\left(E_{\nu_{\mu}\left(\bar{\nu}_{\mu}\right)}\right)$ and the polarization observables $\bar{P}_{L}^{h, l}\left(E_{\nu_{\mu}\left(\bar{\nu}_{\mu}\right)}\right), \bar{P}_{P}^{h, l}\left(E_{\nu_{\mu}\left(\bar{\nu}_{\mu}\right)}\right)$ and $\bar{P}_{T}^{h, l}\left(E_{\nu_{\mu}\left(\bar{\nu}_{\mu}\right)}\right)$ of the hadrons $\left(h=n, p, \Lambda, \Sigma^{0}, \Sigma^{-}\right)$and the

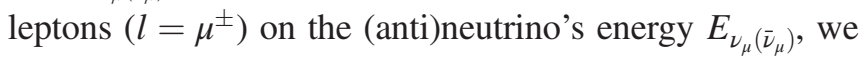
have integrated $d \sigma / d Q^{2}, P_{L}^{h, l}\left(Q^{2}\right), P_{P}^{h, l}\left(Q^{2}\right)$ and $P_{T}^{h, l}\left(Q^{2}\right)$ over $Q^{2}$ and obtained the expressions for $\sigma\left(E_{\nu_{\mu}\left(\bar{\nu}_{\mu}\right)}\right)$ and $\bar{P}_{L, P, T}^{h, l}\left(E_{\nu_{\mu}\left(\bar{\nu}_{\mu}\right)}\right)$ as

$$
\sigma\left(E_{\nu_{\mu}\left(\bar{\nu}_{\mu}\right)}\right)=\int_{Q_{\min }^{2}}^{Q_{\max }^{2}} \frac{d \sigma}{d Q^{2}} d Q^{2},
$$

and

$$
\bar{P}_{L, P, T}^{h, l}\left(E_{\nu_{\mu}\left(\bar{\nu}_{\mu}\right)}\right)=\frac{\int_{Q_{\min }^{2}}^{Q_{\max }^{2}} P_{L, P, T}^{h, l}\left(Q^{2}\right) \frac{d \sigma}{d Q^{2}} d Q^{2}}{\int_{Q_{\min }^{2}}^{Q_{\max }^{2}} \frac{d \sigma}{d Q^{2}} d Q^{2}} .
$$

In Fig. 3, the numerical results are presented for the total cross sections $\sigma\left(E_{\nu_{\mu}}\right)$ for the process $\nu_{\mu}+n \rightarrow \mu^{-}+p$ and the average polarizations $\bar{P}_{L}^{p}\left(E_{\nu_{\mu}}\right)$ and $\bar{P}_{P}^{p}\left(E_{\nu_{\mu}}\right)$ for the outgoing proton as a function of neutrino energy for the
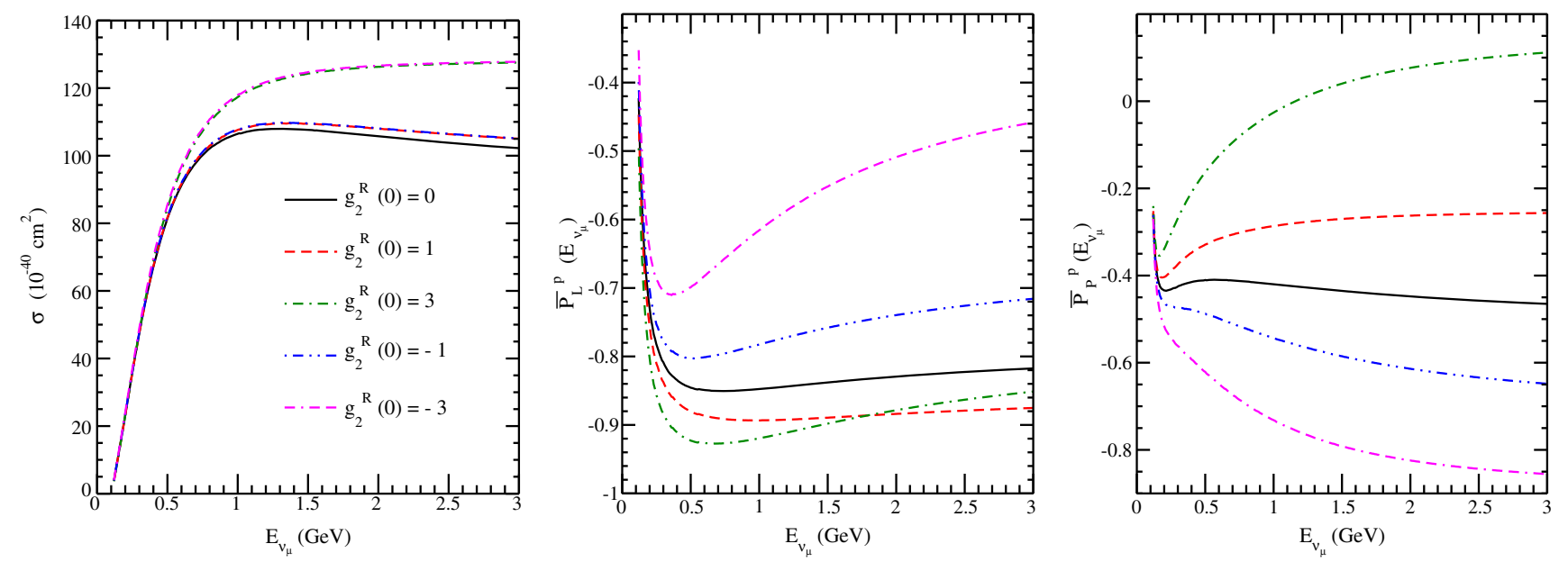

FIG. 3. $\sigma$ vs $E_{\nu_{\mu}}$ (left panel) for the process $\nu_{\mu}+n \rightarrow \mu^{-}+p, \bar{P}_{L}^{p}\left(E_{\nu_{\mu}}\right)$ vs $E_{\nu_{\mu}}$ (middle panel) and $\bar{P}_{P}^{p}\left(E_{\nu_{\mu}}\right)$ vs $E_{\nu_{\mu}}$ (right panel), for the polarized proton in the final state, at the different values of $g_{2}^{R}(0)$ viz. $g_{2}^{R}(0)=0$ (solid line), 1 (dashed line), 3 (dashed-dotted line), -1 (double-dotted-dashed line) and -3 (double-dashed-dotted line). 
various values of $g_{2}^{n p}(0)$ taking it to be real, i.e., $g_{2}^{n p}(0)=\left|g_{2}^{R}(0)\right|=0, \quad 1$ and 3 . We find that $\sigma\left(E_{\nu_{\mu}}\right)$ increases with the neutrino energy for all values of $g_{2}^{R}(0)$. In the case of $g_{2}^{R}(0) \neq 0$, there is a further enhancement in the cross section which increases with $g_{2}^{R}(0)$ and the increase is significant only when $\left|g_{2}^{R}(0)\right| \geq 1$. The increase in $\sigma\left(E_{\nu_{\mu}}\right)$ is almost the same for the positive as well as the negative values of $g_{2}^{R}(0)$. However, the average values of the longitudinal and perpendicular components of the polarization $\bar{P}_{L}^{p}\left(E_{\nu_{\mu}}\right)$ and $\bar{P}_{P}^{p}\left(E_{\nu_{\mu}}\right)$ are negative for all the values of $\left|g_{2}^{R}(0)\right|$ in the range $0-3$ in the entire energy range of $E_{\nu_{\mu}}$ considered here. The absolute value of $\bar{P}_{L}^{p}\left(E_{\nu_{\mu}}\right)$ shows similar trend for the positive as well as the negative values of $g_{2}^{R}(0)$; i.e., it decreases with the increase in energy. The absolute value of $\bar{P}_{P}^{p}\left(E_{\nu_{\mu}}\right)$ decreases with energy for $g_{2}^{R}(0)>0$ and increases for $g_{2}^{R}(0) \leq 0$.
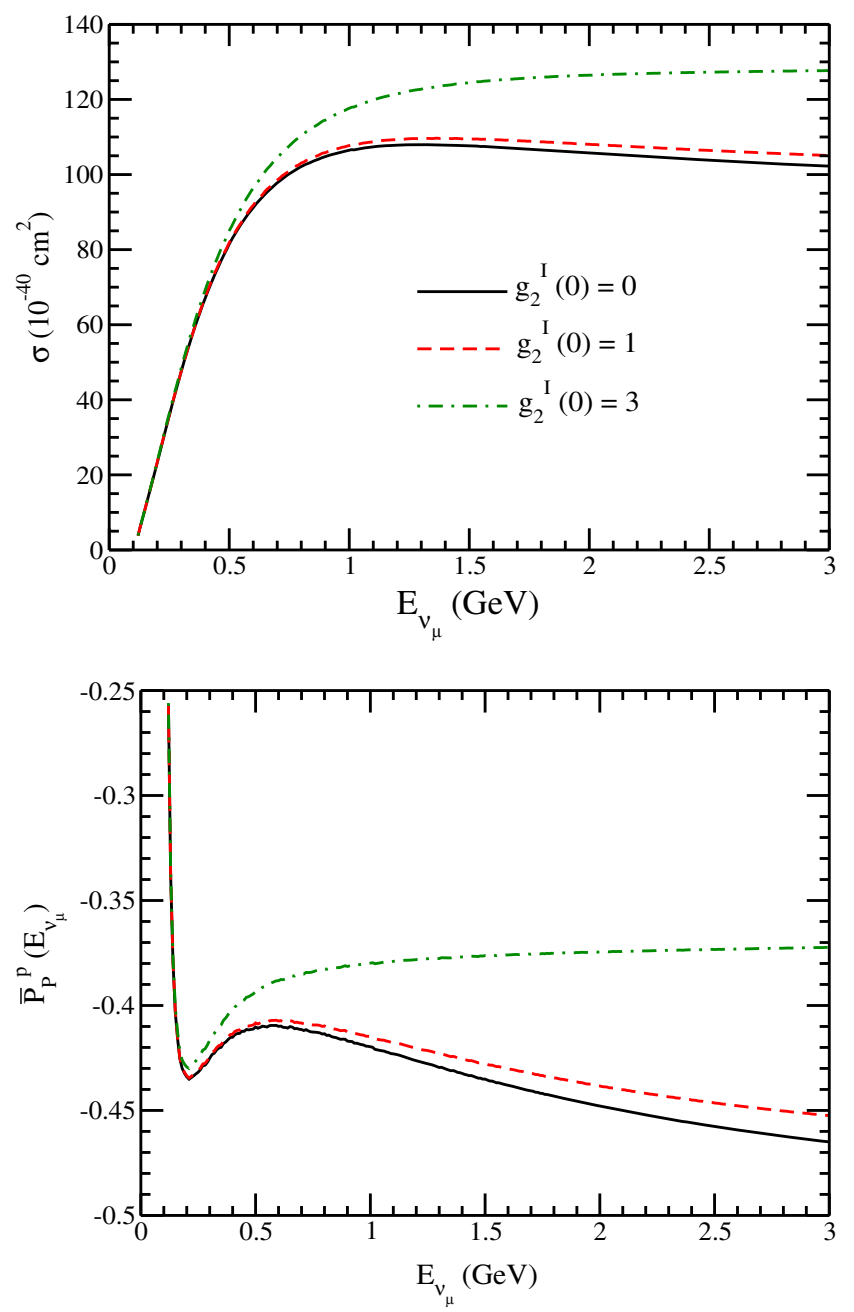

In Fig. 4, we present the results for $\sigma\left(E_{\nu_{\mu}}\right)$ and $\bar{P}_{L, P, T}\left(E_{\nu_{\mu}}\right)$ as a function of neutrino energy $E_{\nu_{\mu}}$ for the various values of $g_{2}^{n p}(0)$ taken to be purely imaginary, i.e., $g_{2}^{n p}(0)=g_{2}^{I}(0)=0,1$ and 3 . We find that the value of $\sigma\left(E_{\nu_{\mu}}\right)$ increases with energy for all values of $g_{2}^{I}(0)$. The effect of $g_{2}^{I}(0) \neq 0$ is to further increase the cross section and the increase is significant only for $\left|g_{2}^{I}(0)\right| \geq 1$. The longitudinal $\bar{P}_{L}^{p}\left(E_{\nu_{\mu}}\right)$ and perpendicular $\bar{P}_{P}^{p}\left(E_{\nu_{\mu}}\right)$ components of the proton polarization are negative and their absolute values decrease with the increase in $g_{2}^{I}(0) \geq 0$. The results for $\bar{P}_{L}^{p}\left(E_{\nu_{\mu}}\right)$ and $\bar{P}_{P}^{p}\left(E_{\nu_{\mu}}\right)$ remain unchanged when the negative values of $g_{2}^{I}(0)$ are taken as they depend on $\operatorname{Reg}_{2}\left(Q^{2}\right)$ and $\left|g_{2}\left(Q^{2}\right)\right|^{2}$ (see Eq. (A1) and (A5)). The transverse component of the proton polarization which violates TRI is now nonvanishing and is positive for $g_{2}^{I}(0) \geq 0$ and increases with energy. Even for $g_{2}^{I}(0)=1$, it could be $\sim 10 \%$ for $E_{\nu_{\mu}} \sim 1 \mathrm{GeV}$ and becomes larger for
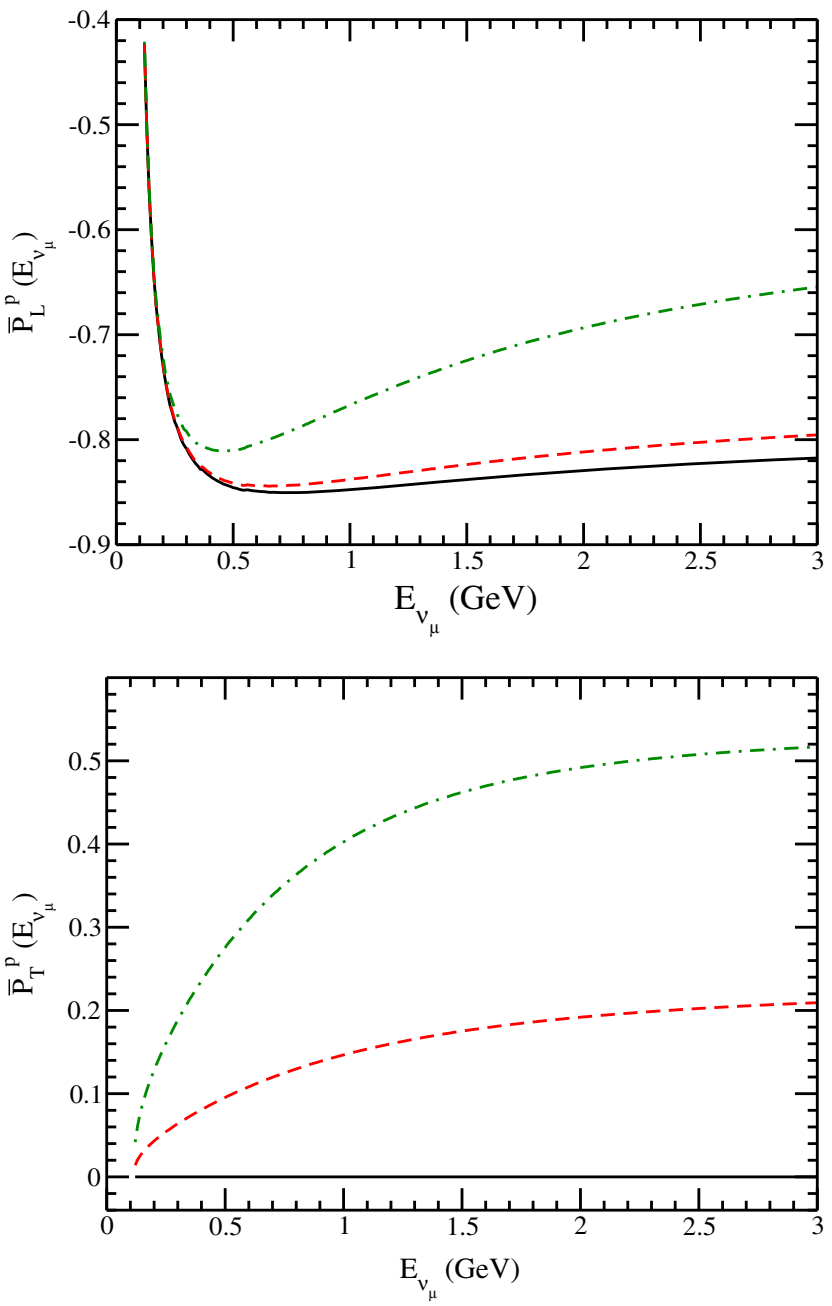

FIG. 4. $\sigma$ vs $E_{\nu_{\mu}}$ (upper left panel) for the process $\nu_{\mu}+n \rightarrow \mu^{-}+p, \bar{P}_{L}^{p}\left(E_{\nu_{\mu}}\right)$ vs $E_{\nu_{\mu}}$ (upper right panel), $\bar{P}_{P}^{p}\left(E_{\nu_{\mu}}\right)$ vs $E_{\nu_{\mu}}$ (lower left panel) and $\bar{P}_{T}^{p}\left(E_{\nu_{\mu}}\right)$ vs $E_{\nu_{\mu}}$ (lower right panel), for the polarized proton in the final state, at the different values of $g_{2}^{I}(0)$ viz. $g_{2}^{I}(0)=0$ (solid line), 1 (dashed line) and 3 (dashed-dotted line). 

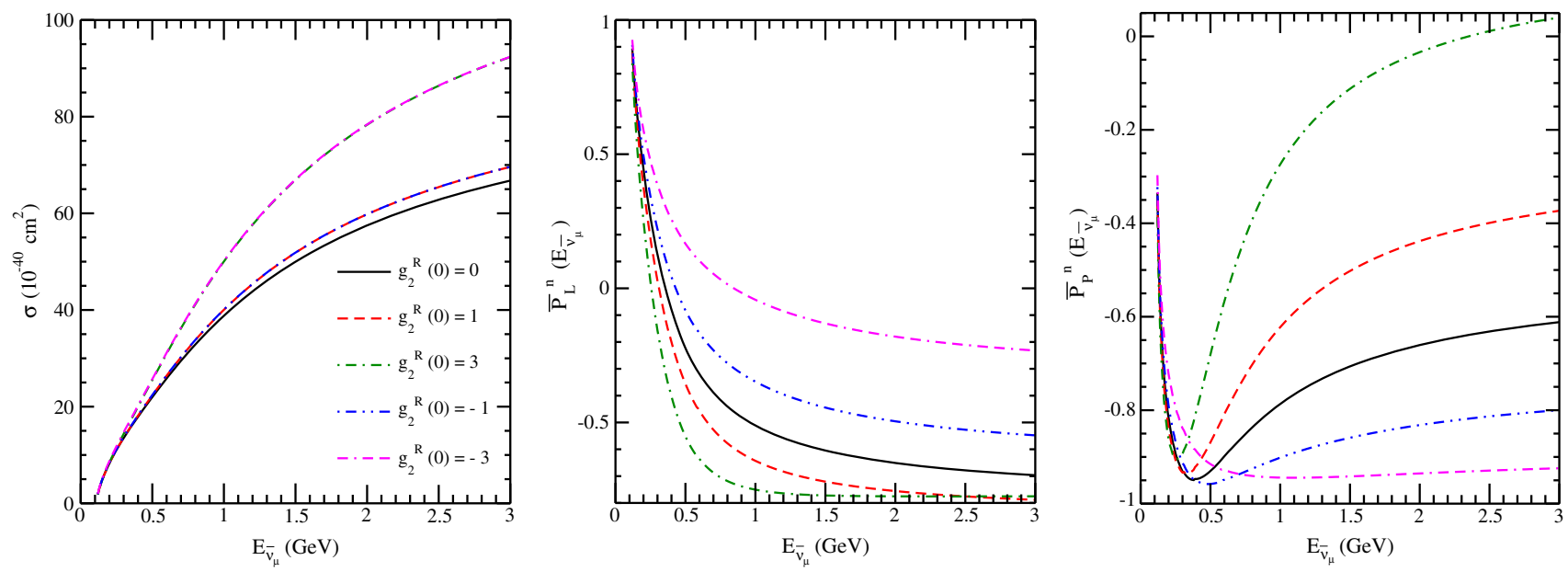

FIG. 5. $\sigma$ vs. $E_{\bar{\nu}_{\mu}}$ (left panel) for the process $\bar{\nu}_{\mu}+p \rightarrow \mu^{+}+n, \bar{P}_{L}^{n}\left(E_{\bar{\nu}_{\mu}}\right)$ vs. $E_{\bar{\nu}_{\mu}}$ (middle panel) and $\bar{P}_{P}^{n}\left(E_{\bar{\nu}_{\mu}}\right)$ vs. $E_{\bar{\nu}_{\mu}}$ (right panel), for the polarized neutron in the final state. Lines and points have the same meaning as in Fig. 3.

$g_{2}^{I}(0) \geq 1$. In the case of $g_{2}^{I}(0)<0$, the transverse component of the polarization $\bar{P}_{T}^{p}\left(E_{\nu_{\mu}}\right)$ just changes sign but the magnitude remains the same, and therefore, the results have not been depicted in the figures.

The results for the total cross section for the reaction $\bar{\nu}_{\mu}+p \rightarrow \mu^{+}+n$, and the various components of the polarization for the neutron are presented in Fig. 5 for $g_{2}^{R}(0)$ by taking the different values of $g_{2}^{R}(0)$ viz. $0, \pm 1$ and \pm 3 . We see that with the increase in the value of $\left|g_{2}^{R}(0)\right|$, the total cross section $\sigma\left(E_{\bar{\nu}_{\mu}}\right)$ increases with energy for all values of $g_{2}^{R}(0)$. In the case of $g_{2}^{R}(0) \neq 0$, there is further increase in the cross section which is almost the same for $g_{2}^{R}(0)>0$ and $g_{2}^{R}(0)<0$. The longitudinal component of the polarization $\bar{P}_{L}^{n}\left(E_{\bar{\nu}_{\mu}}\right)$ is positive for the smaller values of energy $\left(E_{\bar{\nu}_{\mu}}<0.3 \mathrm{GeV}\right)$ and then becomes negative for all values of $g_{2}^{R}(0)$. In the case of nonzero values of $g_{2}^{R}(0)$, the absolute value of $\bar{P}_{L}^{n}\left(E_{\bar{\nu}_{\mu}}\right)$ increases with the energy and the increase is more (less) for the positive (negative) values of $g_{2}^{R}(0)$ as compared to $g_{2}^{R}(0)=0$. In the case of the perpendicular component of the polarization, $\bar{P}_{P}^{n}\left(E_{\bar{\nu}_{\mu}}\right)$ is always negative and its absolute value increases with energy at low energies $E_{\bar{\nu}_{\mu}} \leq 0.3 \mathrm{GeV}$ and then decreases with energy for all $g_{2}^{R}(0)$. In the case of nonzero values of $g_{2}^{R}(0)$, the absolute value of $\bar{P}_{P}^{n}\left(E_{\bar{\nu}_{\mu}}\right)$ still decreases but the decrease is more (less) for positive (negative) values of $g_{2}^{R}(0)$ as compared to $g_{2}^{R}(0)=0$.

In Fig. 6, we have shown the results for $\sigma\left(E_{\bar{\nu}_{\mu}}\right)$ and $\bar{P}_{L, P, T}^{n}\left(E_{\bar{\nu}_{\mu}}\right)$ for various values of $g_{2}^{n p}(0)$ taken to be purely imaginary, i.e., $g_{2}^{I}(0)=0,1$ and 3. The results for $\sigma\left(E_{\bar{\nu}_{\mu}}\right)$, $\bar{P}_{L}^{n}\left(E_{\bar{\nu}_{\mu}}\right)$ and $\bar{P}_{P}^{n}\left(E_{\bar{\nu}_{\mu}}\right)$ are qualitatively similar to Fig. 5. The average value of the transverse component of the polarization $\bar{P}_{T}^{n}\left(E_{\bar{\nu}_{\mu}}\right)$ is nonzero and qualitatively similar to the case of $\bar{P}_{T}^{p}\left(E_{\nu_{\mu}}\right)$ for proton shown in Fig. 4.
We notice from Figs. 3-6 that the results for $\sigma\left(E_{\nu_{\mu}}\left(\bar{\nu}_{\mu}\right)\right)$ increases in the presence of the SCC with (without) TRI, i.e., $g_{2}^{R}(0)\left(g_{2}^{I}(0)\right) \neq 0$. The increase is almost the same for the real as well as the imaginary values of $g_{2}(0)$. It is also well known that with the increase in the value of the axial dipole mass $M_{A}$, the cross section increases. For example, with $20 \%$ increase in the value of $M_{A}$, i.e., from 1 to $1.2 \mathrm{GeV}$, the cross section increases by about $20 \%$. A higher value of $M_{A}$ from the world average value (i.e., $M_{A}=1.026 \mathrm{GeV}$ ) is suggested from (anti)neutrino scattering experiments in the higher energy region $[85,86]$. It is, therefore, possible that a nonzero value of $g_{2}^{R}(0)$ and/or $g_{2}^{I}(0)$, i.e., the existence of the SCC with or without TRI, may lead to a smaller value of $M_{A}$. Keeping this in mind, in Fig. 7, we have studied the dependence of the cross section on $M_{A}$ with or without the presence of $g_{2}^{R}(0)$ or $g_{2}^{I}(0)$. It may be observed from the figure that, in the case of neutrino induced process, i.e., $\nu_{\mu}+n \rightarrow \mu^{-}+p$, the results obtained by taking $M_{A}=1.1 \mathrm{GeV}$ and $g_{2}^{R}(0)=0$ are comparable to the results obtained with $M_{A}=$ $1.026 \mathrm{GeV}$ and $g_{2}^{R}(0)=2$, whereas the results obtained by taking $M_{A}=1.2 \mathrm{GeV}$ and $g_{2}^{R}(0)=0$ are comparable to the results obtained using $M_{A}=1.026 \mathrm{GeV}$ and $g_{2}^{R}(0)=3$. While in the case of antineutrino induced process, i.e., $\bar{\nu}_{\mu}+p \rightarrow \mu^{+}+n$, the results obtained by taking $M_{A}=1.1 \mathrm{GeV}$ and $g_{2}^{R}(0)=0$ are comparable to the results obtained with $M_{A}=1.026 \mathrm{GeV}$ and $g_{2}^{R}(0)=1$ whereas the results obtained by taking $M_{A}=1.2 \mathrm{GeV}$ and $g_{2}^{R}(0)=0$ are slightly lower than the results obtained using $M_{A}=1.026 \mathrm{GeV}$ and $g_{2}^{R}(0)=2$. Thus, a higher value of $\sigma\left(E_{\bar{\nu}_{\mu}}\right)$ may be obtained by either taking a nonzero value of $g_{2}^{n p}(0)$ or increasing the value of $M_{A}$. Furthermore, the cross section measurements may give information only about the nonzero value of $g_{2}^{n p}(0)$ irrespective of the nature of the SCC current, i.e., with or without TRI. One may 

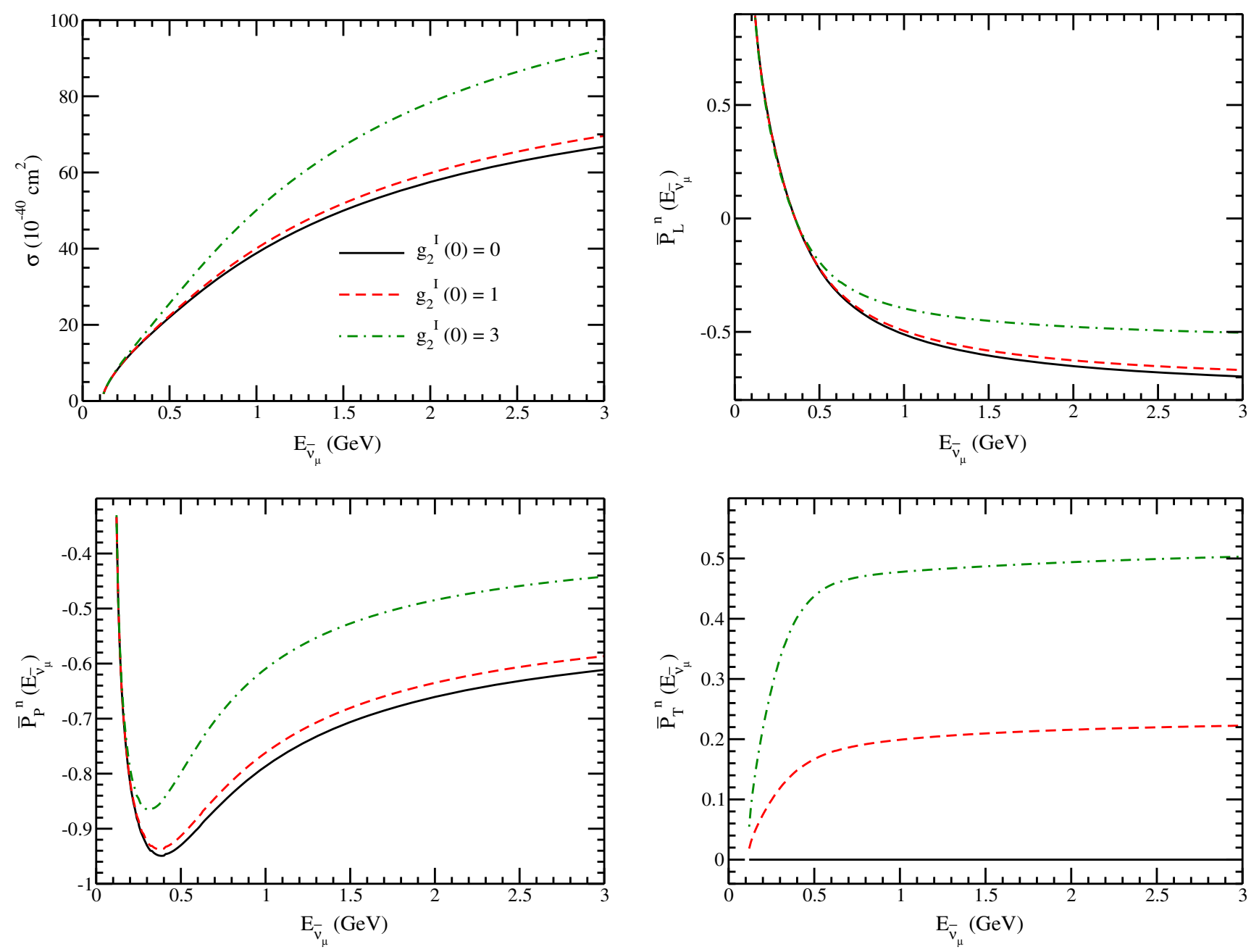

FIG. 6. $\sigma$ vs. $E_{\bar{\nu}_{\mu}}$ (upper left panel) for the process $\bar{\nu}_{\mu}+p \rightarrow \mu^{+}+n, \bar{P}_{L}^{n}\left(E_{\bar{\nu}_{\mu}}\right)$ vs. $E_{\bar{\nu}_{\mu}}$ (upper right panel), $\bar{P}_{P}^{n}\left(E_{\bar{\nu}_{\mu}}\right)$ vs. $E_{\bar{\nu}_{\mu}}($ lower left panel) and $\bar{P}_{T}^{n}\left(E_{\bar{\nu}_{\mu}}\right)$ vs. $E_{\bar{\nu}_{\mu}}$ (lower right panel), for the polarized neutron in the final state. Lines and points have the same meaning as in Fig. 4.
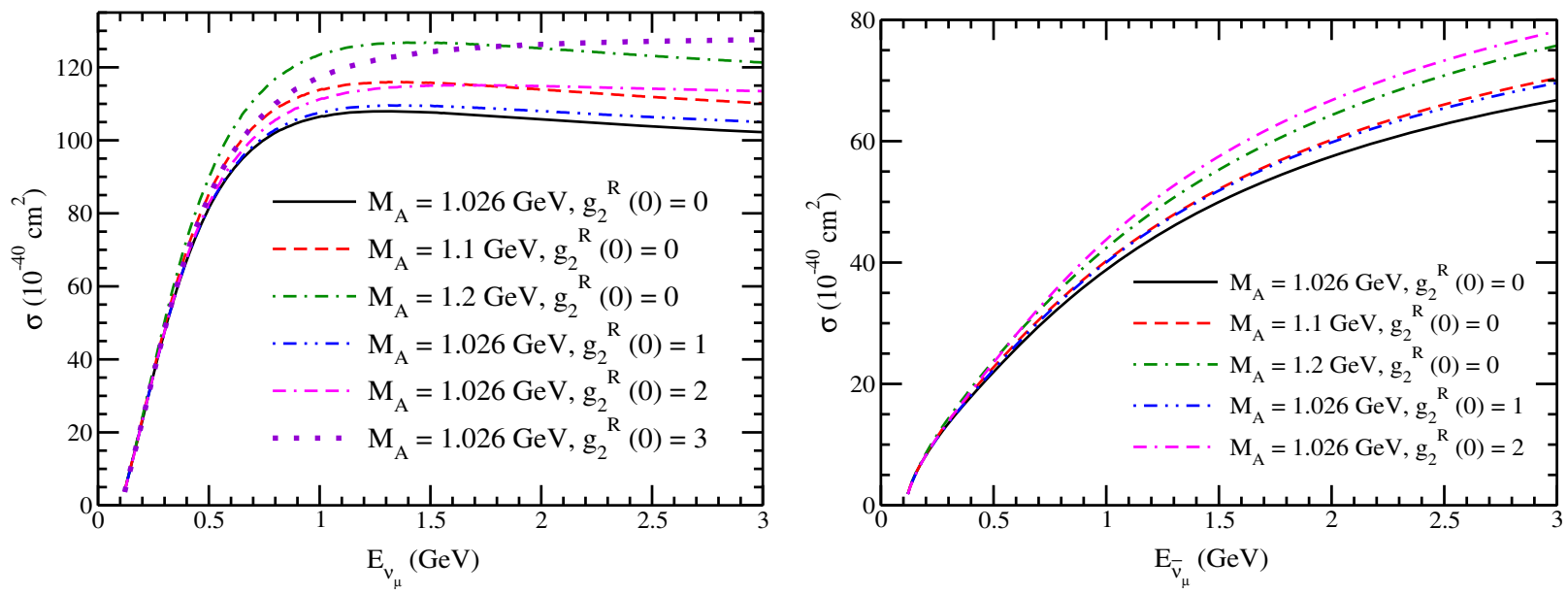

FIG. 7. $\sigma$ vs. $E_{\nu_{\mu}\left(\bar{\nu}_{\mu}\right)}$ for the process $\nu_{\mu}+n \rightarrow \mu^{-}+p$ (left panel) and $\bar{\nu}_{\mu}+p \rightarrow \mu^{+}+n$ (right panel) for the different combinations of $M_{A}$, and $g_{2}^{R}(0)$ viz. $M_{A}=1.026 \mathrm{GeV}$ and $g_{2}^{R}(0)=0$ (solid line), $M_{A}=1.1 \mathrm{GeV}$ and $g_{2}^{R}(0)=0$ (dashed line), $M_{A}=1.2 \mathrm{GeV}$ and $g_{2}^{R}(0)=0$ (dashed-dotted line), $M_{A}=1.026 \mathrm{GeV}$ and $g_{2}^{R}(0)=1$ (double-dotted-dashed line), $M_{A}=1.026 \mathrm{GeV}$ and $g_{2}^{R}(0)=2$ (double-dashed-dotted line) and $M_{A}=1.026 \mathrm{GeV}$ and $g_{2}^{R}(0)=3$ (dotted line). 

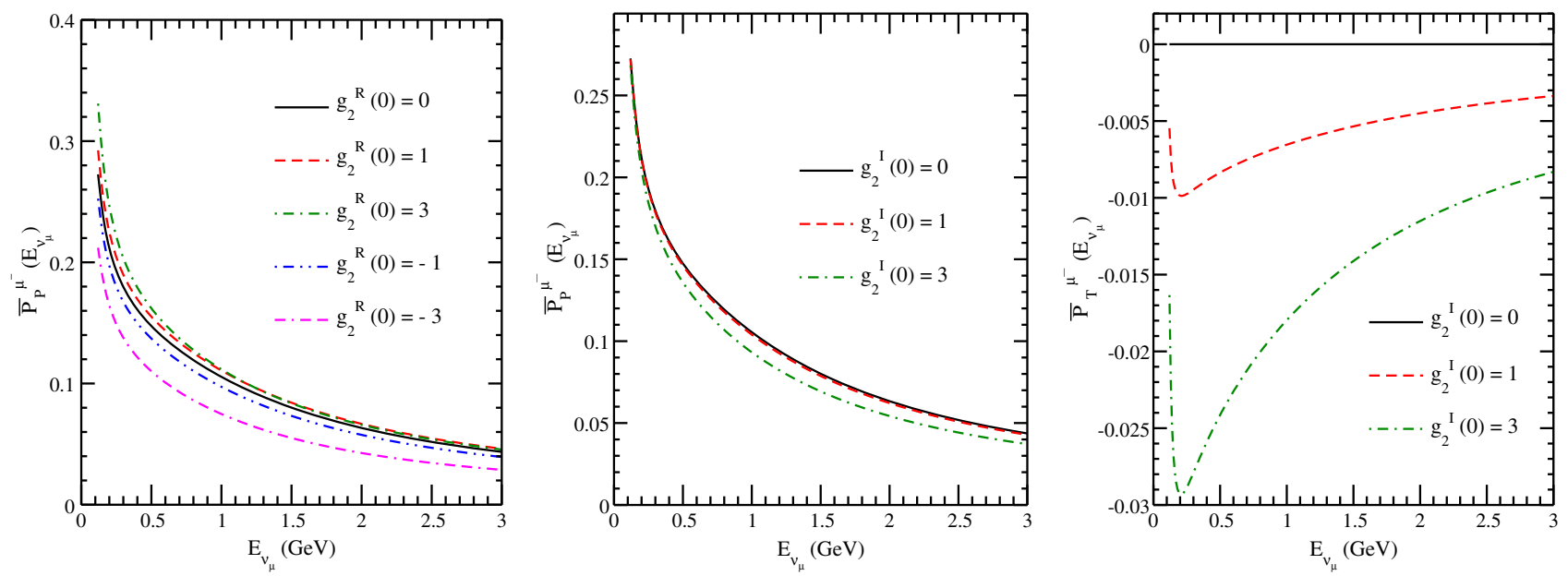

FIG. 8. $\quad \bar{P}_{P}^{\mu^{-}}\left(E_{\nu_{\mu}}\right)$ vs. $E_{\nu_{\mu}}$ for the real values of $g_{2}^{n p}(0)$ (left panel), $\bar{P}_{P}^{\mu^{-}}\left(E_{\nu_{\mu}}\right)$ vs. $E_{\nu_{\mu}}$ for the imaginary values of $g_{2}^{n p}(0)$ (middle panel) and $\bar{P}_{T}^{\mu^{-}}\left(E_{\nu_{\mu}}\right)$ vs. $E_{\nu_{\mu}}$ (right panel) for the process $\nu_{\mu}+n \rightarrow \mu^{-}+p$ at the different values of $g_{2}^{R, I}(0)$ viz. $g_{2}^{R, I}(0)=0$ (solid line), 1 (dashed line), 3 (dashed-dotted line), -1 (double-dotted-dashed line) and -3 (double-dashed-dotted line).

obtain the nature of the SCC by measuring the polarization observables which gives different results with real and imaginary values of $g_{2}^{n p}(0)$ corresponding to the SCC with or without TRI.

In Fig. 8, we have presented the results for the perpendicular and the transverse components of the polarization of the muon produced in the reaction $\nu_{\mu}+n \rightarrow$ $\mu^{-}+p$ for the real as well as the imaginary value of $g_{2}^{n p}(0)$. Since the relativistic muons are known to be longitudinal, therefore, we have presented the results only for $\bar{P}_{P}^{\mu}\left(E_{\nu_{\mu}}\right)$ and $\bar{P}_{T}^{\mu}\left(E_{\nu_{\mu}}\right)$. We find that $\bar{P}_{P}^{\mu}\left(E_{\nu_{\mu}}\right)$ is less sensitive to the values of $g_{2}^{R}(0)$ as well $g_{2}^{I}(0)$ unless $\left|g_{2}^{R}(0)\right|>1$. The transverse component of the polarization $\bar{P}_{T}^{\mu}\left(E_{\nu_{\mu}}\right)$ is mildly sensitive to $g_{2}^{I}(0)$, and the sensitivity increases with the increase in $g_{2}^{I}(0)$.
Similarly in Fig. 9, we have presented the results for the perpendicular and the transverse components of polarization of the muon produced in the reaction $\bar{\nu}_{\mu}+p \rightarrow \mu^{+}+n$ for the real as well as the imaginary values of $g_{2}^{n p}(0)$. We find that in the case of real $g_{2}^{n p}(0)$, $\bar{P}_{P}^{\mu}\left(E_{\bar{\nu}_{\mu}}\right)$ shows appreciable sensitivity towards $g_{2}^{R}(0)$. The results for the positive (negative) values of $g_{2}^{R}(0)$ are less (more) as compared to the results obtained with $g_{2}^{R}(0)=0$. In the case of imaginary $g_{2}^{n p}(0)$, the perpendicular component of the polarization is not very sensitive to $g_{2}^{I}(0)$, whereas the transverse component of polarization is sensitive to $g_{2}^{I}(0)$, and the sensitivity increases with the increase in $g_{2}^{I}(0)$.

In Fig. 10, we have presented the results for the total cross section $\sigma\left(E_{\bar{\nu}_{\mu}}\right)$ for the process $\bar{\nu}_{\mu}+p \rightarrow \mu^{+}+\Lambda$,
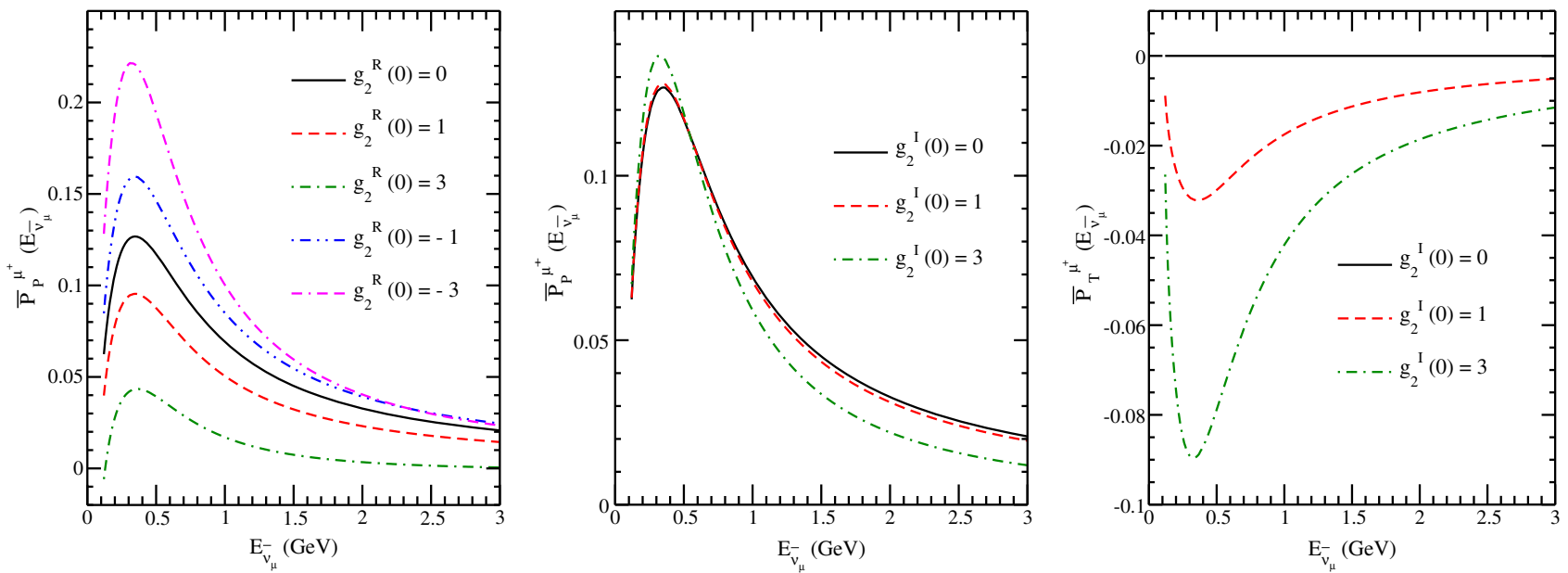

FIG. 9. $\quad \bar{P}_{P}^{\mu^{+}}\left(E_{\bar{\nu}_{\mu}}\right)$ vs. $E_{\bar{\nu}_{\mu}}$ for the real values of $g_{2}^{n p}(0)$ (left panel), $\bar{P}_{P}^{\mu^{+}}\left(E_{\bar{\nu}_{\mu}}\right) v s . E_{\bar{\nu}_{\mu}}$ for the imaginary values of $g_{2}^{n p}(0)$ (middle panel) and $\bar{P}_{T}^{\mu^{+}}\left(E_{\bar{\nu}_{\mu}}\right)$ vs. $E_{\bar{\nu}_{\mu}}$ (right panel) for the process $\bar{\nu}_{\mu}+p \rightarrow \mu^{+}+n$. Lines and points have the same meaning as in Fig. 8 . 

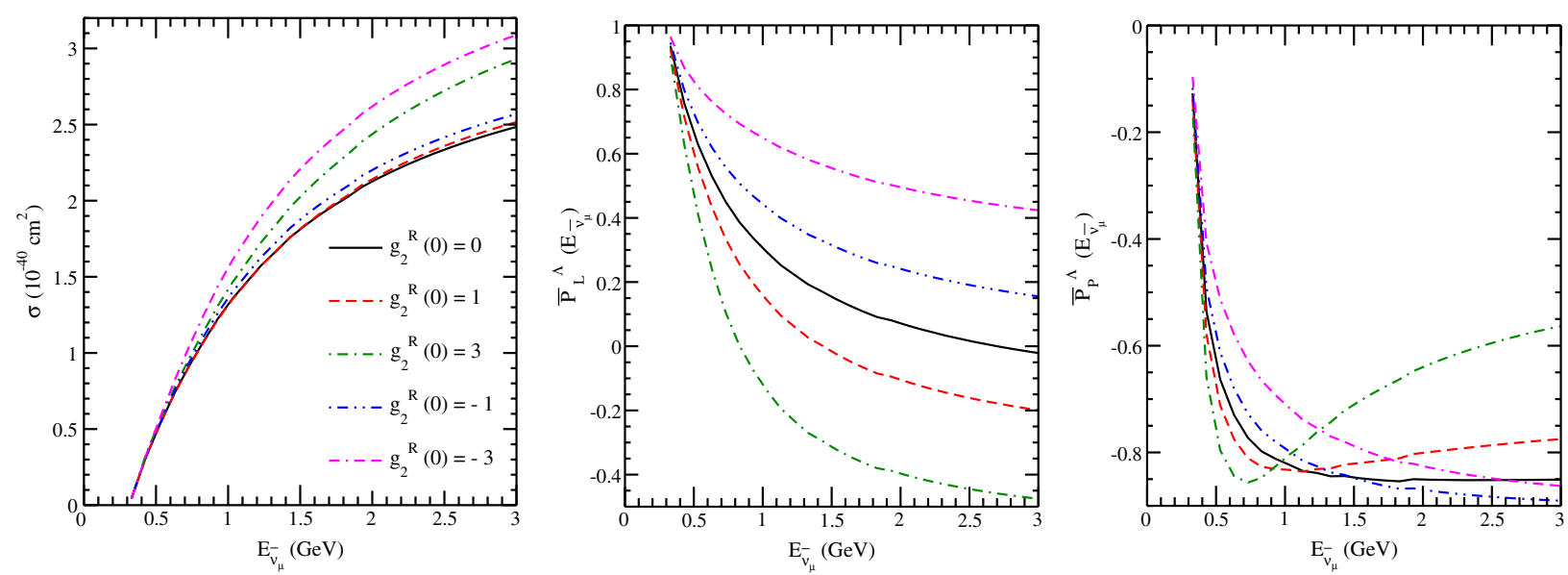

FIG. 10. $\sigma$ vs. $E_{\bar{\nu}_{\mu}}\left(\right.$ left panel) for the process $\bar{\nu}_{\mu}+p \rightarrow \mu^{+}+\Lambda, \bar{P}_{L}^{\Lambda}\left(E_{\bar{\nu}_{\mu}}\right)$ vs. $E_{\bar{\nu}_{\mu}}$ (middle panel) and $\bar{P}_{P}^{\Lambda}\left(E_{\bar{\nu}_{\mu}}\right)$ vs. $E_{\bar{\nu}_{\mu}}$ (right panel), for the polarized lambda in the final state. Lines and points have the same meaning as in Fig. 3.
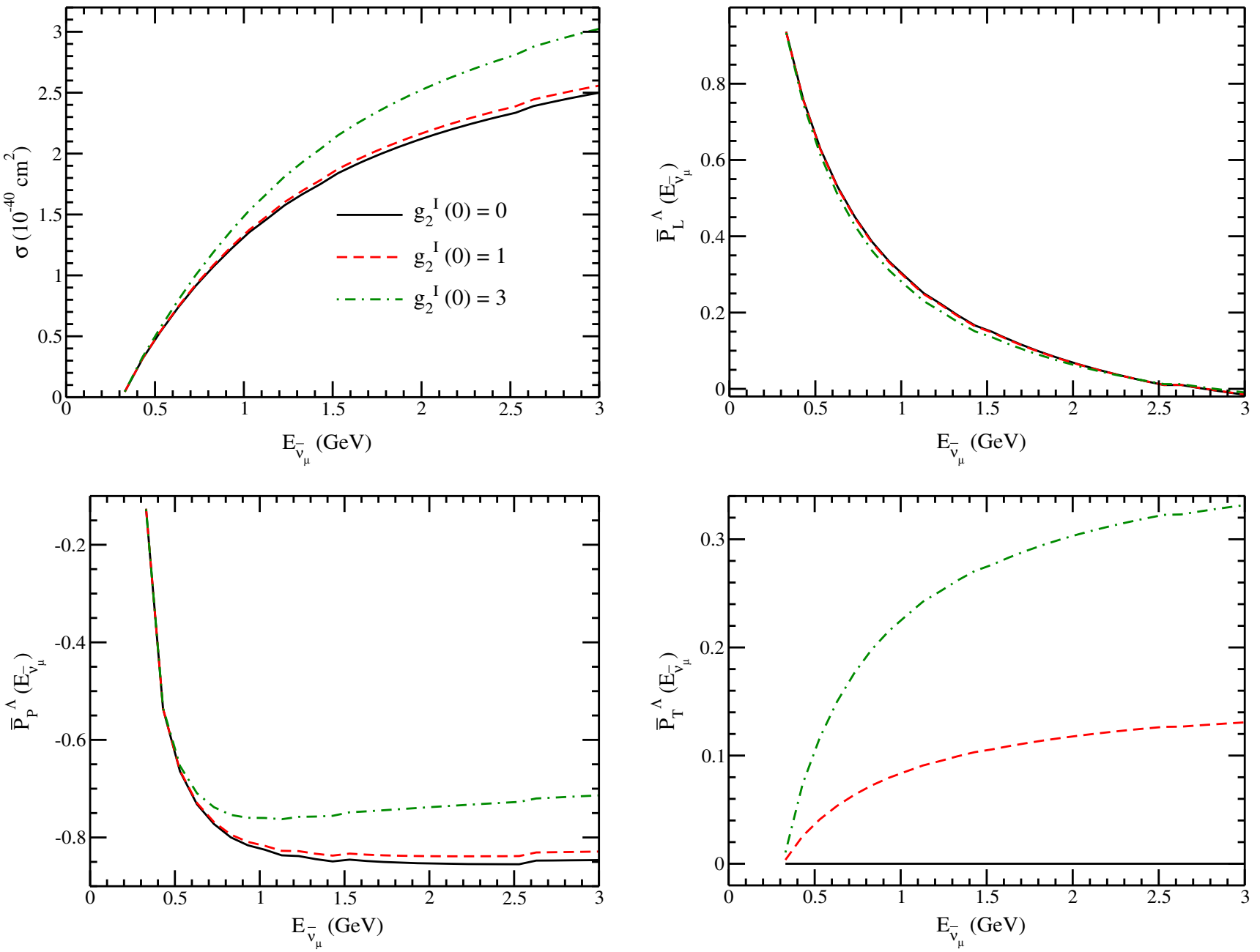

FIG. 11. $\sigma$ vs. $E_{\bar{\nu}_{\mu}}$ (upper left panel) for the process $\bar{\nu}_{\mu}+p \rightarrow \mu^{+}+\Lambda, \bar{P}_{L}^{\Lambda}\left(E_{\bar{\nu}_{\mu}}\right)$ vs. $E_{\bar{\nu}_{\mu}}$ (upper right panel), $\bar{P}_{P}^{\Lambda}\left(E_{\bar{\nu}_{\mu}}\right)$ vs. $E_{\bar{\nu}_{\mu}}($ lower left panel) and $\bar{P}_{T}^{\Lambda}\left(E_{\bar{\nu}_{\mu}}\right)$ vs. $E_{\bar{\nu}_{\mu}}$ (lower right panel), for the polarized lambda in the final state. Lines and points have the same meaning as in Fig. 4. 

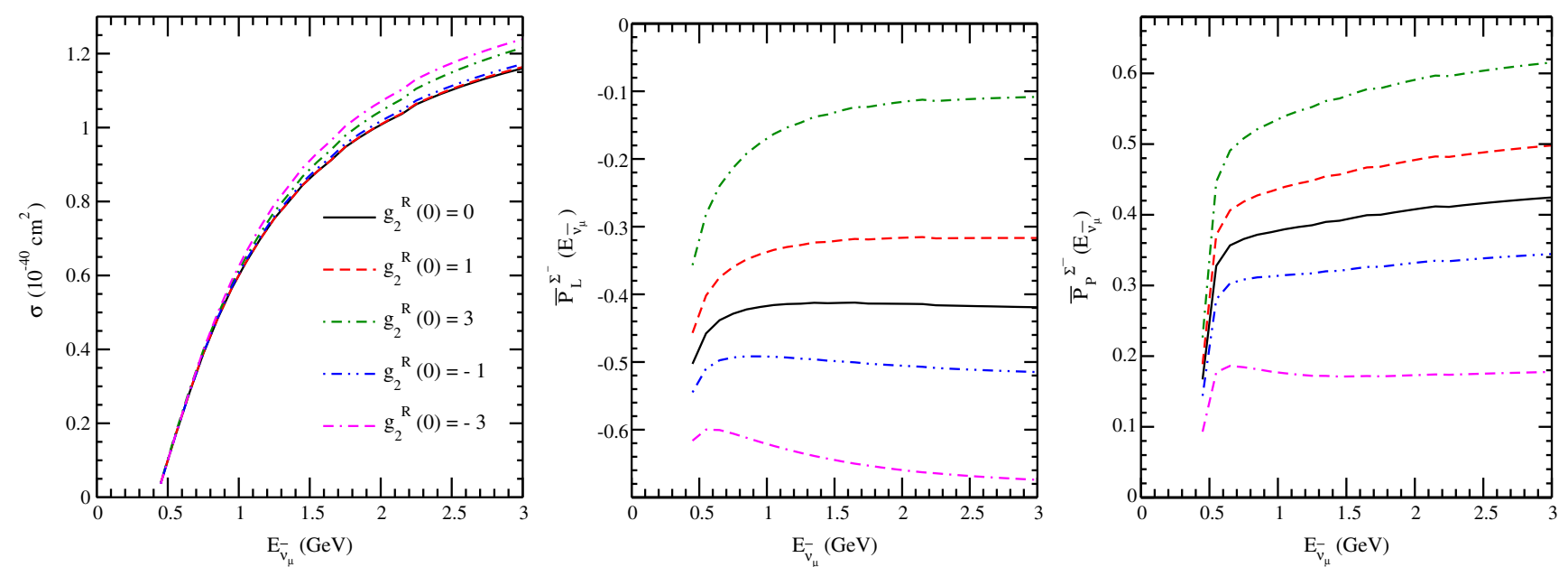

FIG. 12. $\sigma$ vs. $E_{\bar{\nu}_{\mu}}$ (left panel) for the process $\bar{\nu}_{\mu}+n \rightarrow \mu^{+}+\Sigma^{-}, \bar{P}_{L}^{\Sigma^{-}}\left(E_{\bar{\nu}_{\mu}}\right)$ vs. $E_{\bar{\nu}_{\mu}}$ (middle panel) and $\bar{P}_{P}^{\Sigma^{-}}\left(E_{\bar{\nu}_{\mu}}\right)$ vs. $E_{\bar{\nu}_{\mu}}$ (right panel), for a polarized sigma in the final state. Lines and points have the same meaning as in Fig. 3.
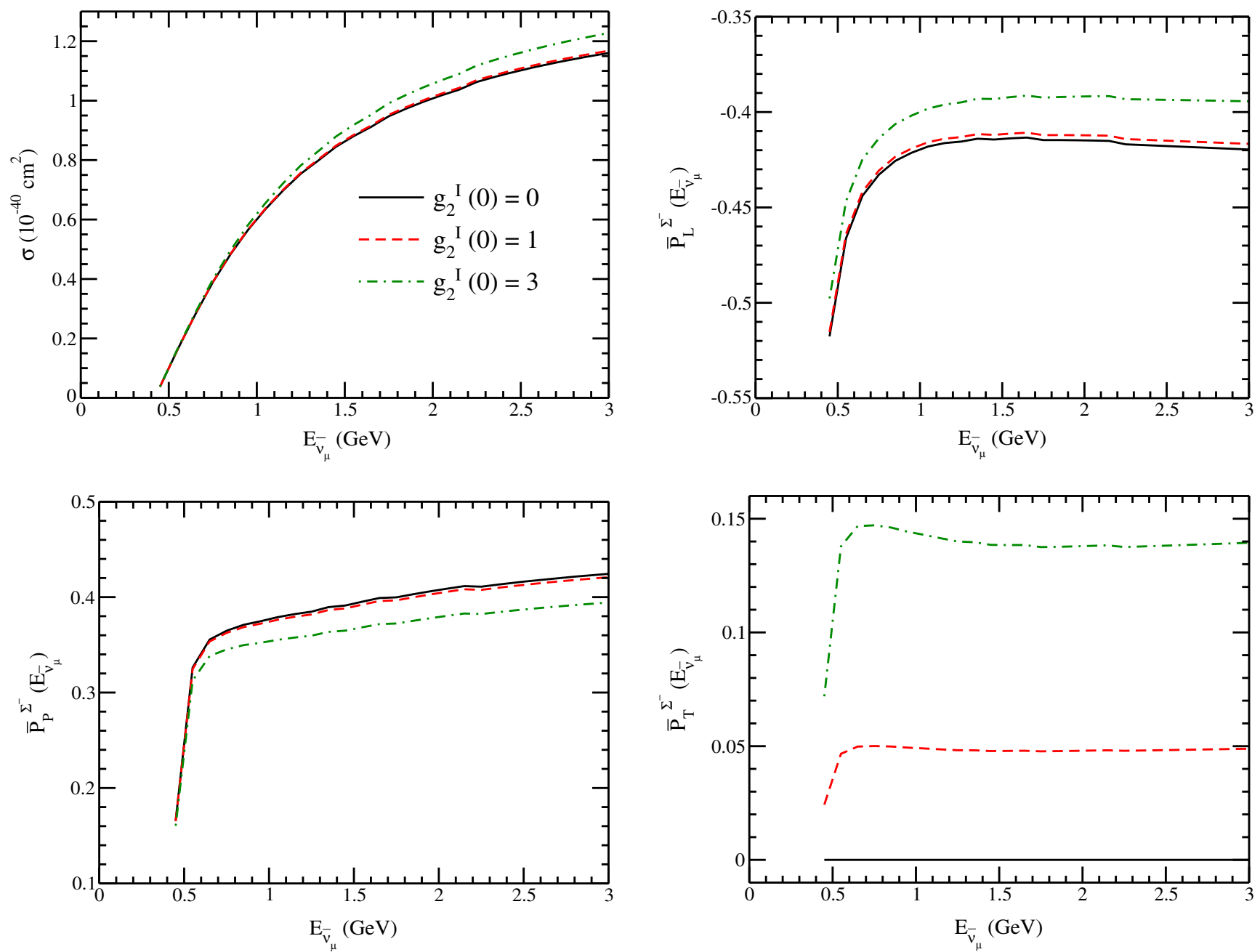

FIG. 13. $\sigma$ vs. $E_{\overline{\bar{\nu}}_{\mu}}$ (upper left panel) for the process $\bar{\nu}_{\mu}+n \rightarrow \mu^{+}+\Sigma^{-}, \bar{P}_{L}^{\Sigma^{-}}\left(E_{\bar{\nu}_{\mu}}\right)$ vs. $E_{\bar{\nu}_{\mu}}$ (upper right panel), $\bar{P}_{P}^{\Sigma^{-}}\left(E_{\bar{\nu}_{\mu}}\right)$ vs. $E_{\bar{\nu}_{\mu}}$ (lower left panel) and $\bar{P}_{T}^{\Sigma^{-}}\left(E_{\bar{\nu}_{\mu}}\right)$ vs. $E_{\bar{\nu}_{\mu}}$ (lower right panel), for a polarized sigma in the final state. Lines and points have the same meaning as in Fig. 4. 

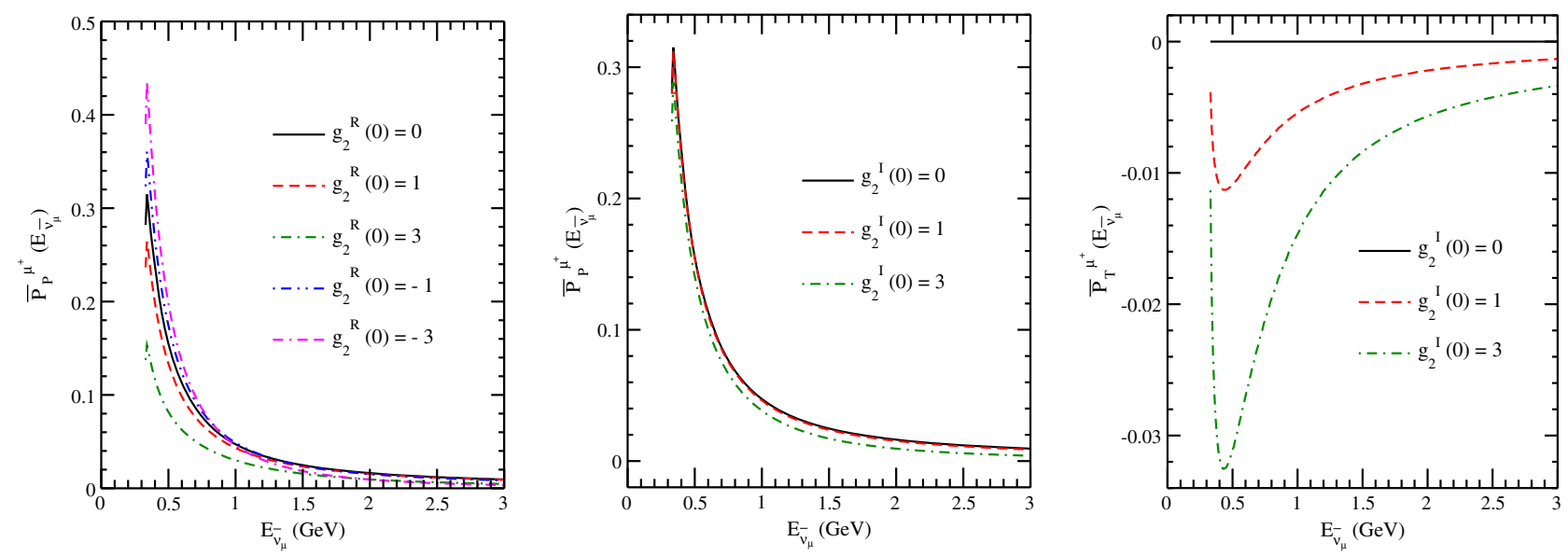

FIG. 14. $\quad \bar{P}_{P}^{\mu^{+}}\left(E_{\bar{\nu}_{\mu}}\right)$ vs. $E_{\bar{\nu}_{\mu}}$ for the real values of $g_{2}^{n p}(0)$ (left panel), $\bar{P}_{P}^{l}\left(E_{\bar{\nu}_{\mu}}\right)$ vs. $E_{\bar{\nu}_{\mu}}$ for the imaginary values of $g_{2}^{n p}(0)$ (middle panel) and $\bar{P}_{T}^{l}\left(E_{\bar{\nu}_{\mu}}\right)$ vs. $E_{\bar{\nu}_{\mu}}$ (right panel) for the process $\bar{\nu}_{\mu}+p \rightarrow \mu^{+}+\Lambda$. Lines and points have the same meaning as in Fig. 8.

and the average polarizations $\bar{P}_{L}^{\Lambda}\left(E_{\bar{\nu}_{\mu}}\right)$ and $\bar{P}_{P}^{\Lambda}\left(E_{\bar{\nu}_{\mu}}\right)$ as a results for the total cross section $\sigma\left(E_{\bar{\nu}_{\mu}}\right)$, and the average function of $E_{\bar{\nu}_{\mu}}$ for the polarized $\Lambda$ at the different polarizations $\bar{P}_{L}^{\Lambda}\left(E_{\bar{\nu}_{\mu}}\right), \bar{P}_{P}^{\Lambda}\left(E_{\bar{\nu}_{\mu}}\right)$ and $\bar{P}_{T}^{\Lambda}\left(E_{\bar{\nu}_{\mu}}\right)$ are presented values of $g_{2}^{R}(0)=0, \pm 1$ and \pm 3 and in Fig. 11, the for the different values of $g_{2}^{I}(0)=0,1$ and 3 . We see that
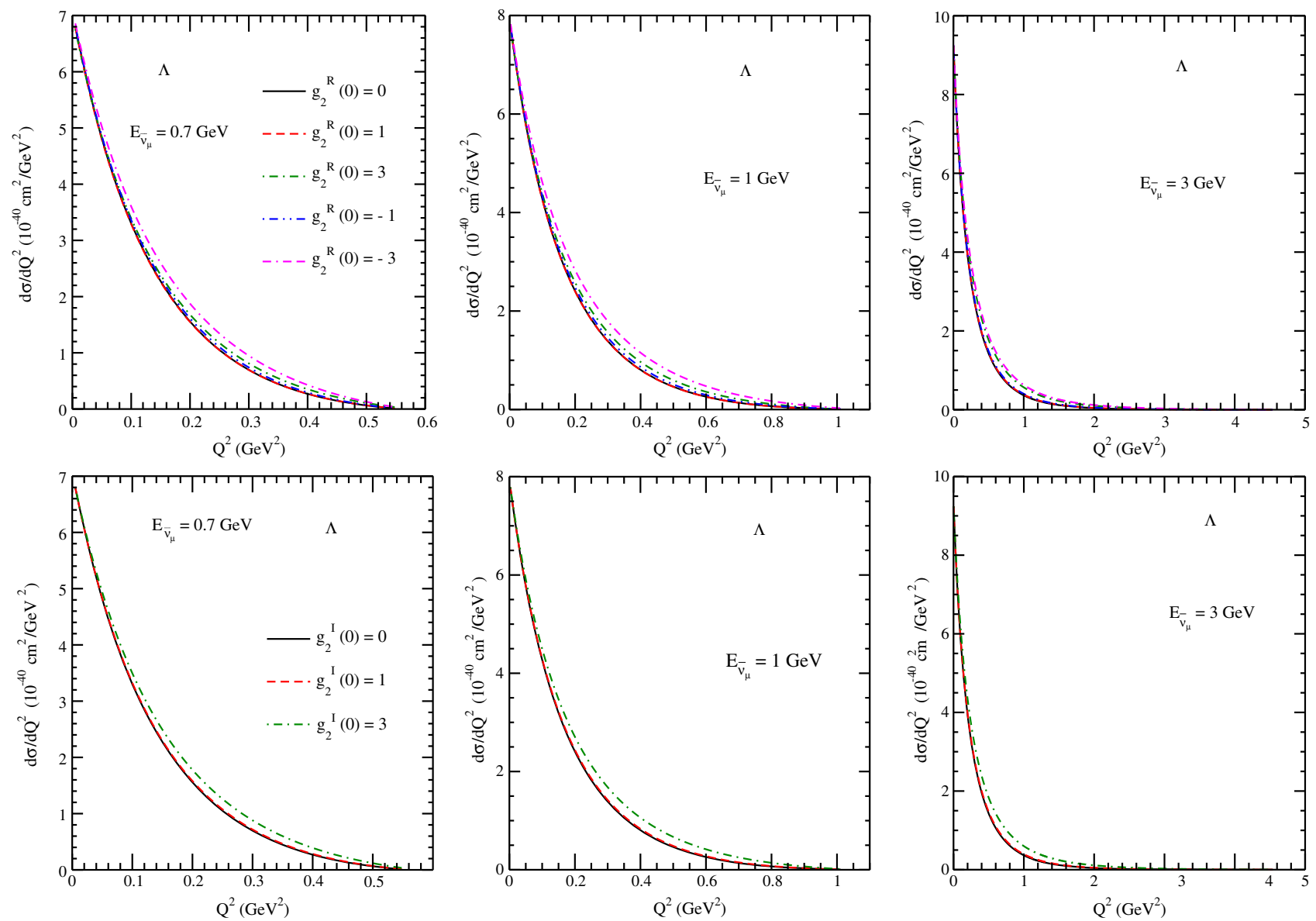

FIG. 15. $d \sigma / d Q^{2}$ for the process $\bar{\nu}_{\mu}+p \rightarrow \mu^{+}+\Lambda$ at the different values of the incoming antineutrino energy, $E_{\bar{\nu}_{\mu}}=0.7 \mathrm{GeV}$ (left panel), $1 \mathrm{GeV}$ (middle panel) and $3 \mathrm{GeV}$ (right panel). The upper panel represents the results with $g_{2}^{R}(0)=0$ (solid line), 1 (dashed line), 3 (dashed-dotted line), -1 (double-dotted-dashed line) and -3 (double-dashed-dotted line) and the lower panel represents the results with $g_{2}^{I}(0)=0$ (solid line), 1 (dashed line) and 3 (dashed-dotted line). 

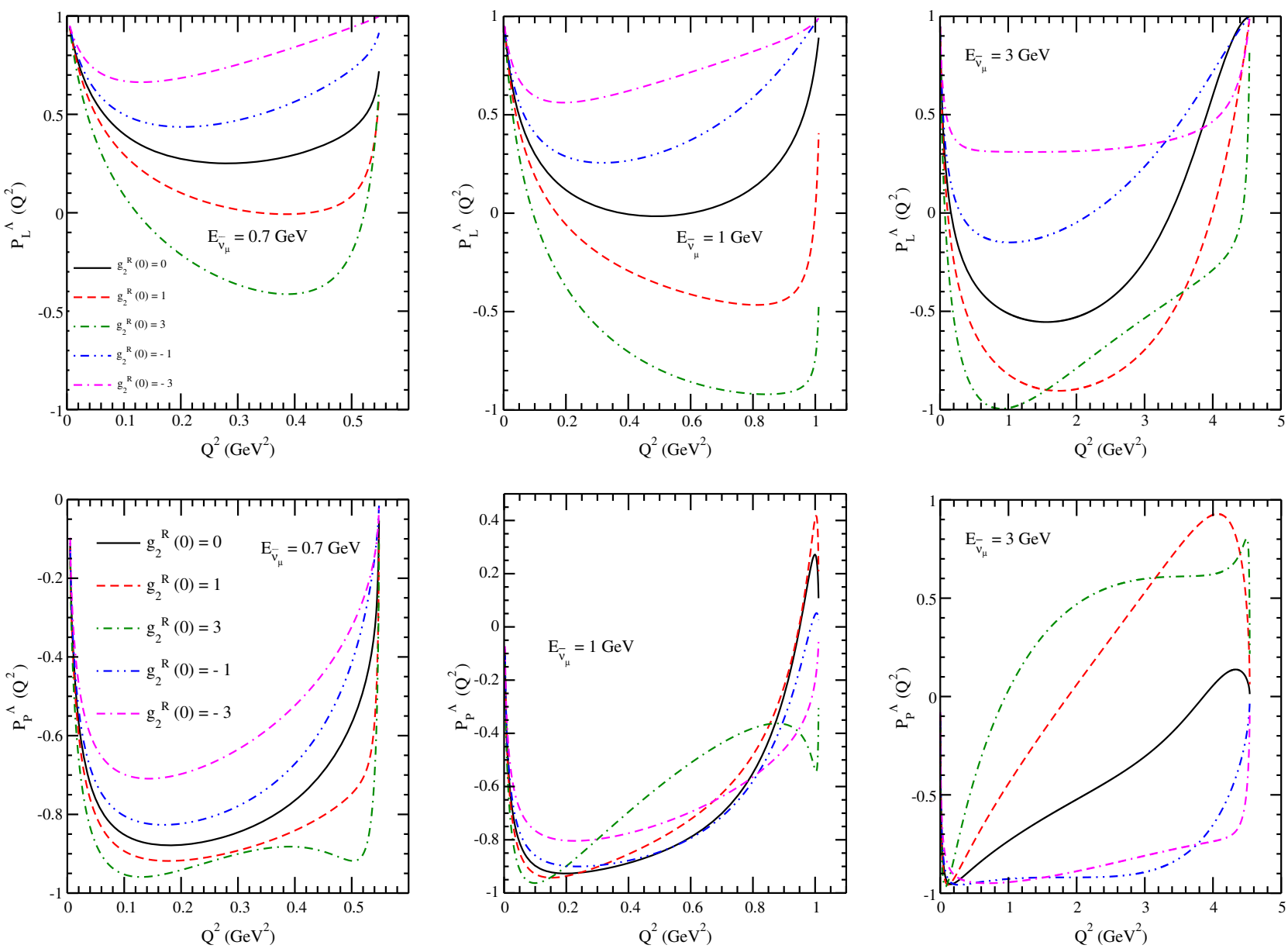

FIG. 16. $P_{L}^{\Lambda}\left(Q^{2}\right)$ vs. $Q^{2}$ (upper panel) and $P_{P}^{\Lambda}\left(Q^{2}\right)$ vs. $Q^{2}$ (lower panel) for the process $\bar{\nu}_{\mu}+p \rightarrow \mu^{+}+\Lambda$ at the different values of the incoming antineutrino energy, $E_{\bar{\nu}_{\mu}}=0.7 \mathrm{GeV}$ (left panel), $1 \mathrm{GeV}$ (middle panel) and $3 \mathrm{GeV}$ (right panel). Lines and points have the same meaning as in Fig. 3.

the results for $\bar{P}_{L, P, T}^{\Lambda}\left(E_{\bar{\nu}_{\mu}}\right)$ are qualitatively similar to the results for neutron in the reaction $\bar{\nu}_{\mu}+p \rightarrow \mu^{+}+n$ but quantitatively they are smaller. However, in the case of the total cross section, unlike the neutron production cross section in the reaction $\bar{\nu}_{\mu}+p \rightarrow \mu^{+}+n$, we find that the positive and negative values of $g_{2}^{R}(0)$ give different values of the total cross section $\sigma\left(E_{\bar{\nu}_{\mu}}\right)$. The results with $g_{2}^{R}(0)<0$ are higher than the results obtained with $g_{2}^{R}(0)>0$.

Similarly, in Figs. 12 and 13, we present the results for $\sigma\left(E_{\bar{\nu}_{\mu}}\right)$ for the reaction $\bar{\nu}_{\mu}+p \rightarrow \mu^{+}+\Sigma^{-}$and $\bar{P}_{L, P, T}^{\Sigma^{-}}\left(E_{\bar{\nu}_{\mu}}\right)$ for the polarized $\Sigma^{-}$in the final state for $\left|g_{2}^{R}(0)\right|$ and $g_{2}^{I}(0)$ in the range $0-3$. We see that while the results for $\sigma\left(E_{\bar{\nu}_{\mu}}\right)$ are qualitatively similar to the results for $\Lambda$ production in the reaction $\bar{\nu}_{\mu}+p \rightarrow \mu^{+}+\Lambda$, the results for $\bar{P}_{L, P, T}^{\Sigma^{-}}\left(E_{\bar{\nu}_{\mu}}\right)$ are qualitatively different from the results for $\bar{P}_{L, P, T}^{\Lambda}\left(E_{\bar{\nu}_{\mu}}\right)$. It should be noted that results for $\sigma^{\Sigma^{0}}\left(E_{\bar{\nu}_{\mu}}\right)$ and $\bar{P}_{L, P, T}^{\Sigma^{0}}\left(E_{\bar{\nu}_{\mu}}\right)$ in the reaction $\bar{\nu}_{\mu}+p \rightarrow \mu^{+}+\Sigma^{0}$ will be similar to $\Sigma^{-}$ production due to the $\mathrm{SU}(3)$ Clebsch Gordan coefficients shown in Table I, and the numerical results for this reaction are not presented here.

In Fig. 14, we present the results for the perpendicular and transverse polarization of muons for the various values of $g_{2}^{R}(0)$ and $g_{2}^{I}(0)$ in the case of $\Lambda$ production. We see that while $\bar{P}_{P}^{\mu}\left(E_{\bar{\nu}_{\mu}}\right)$ is not very sensitive to the value of $g_{2}^{R}(0)$ and $g_{2}^{I}(0)$ unless the value of $g_{2}^{R}(0)$ is much greater than one, i.e., $g_{2}^{R}(0) \geq 1$. However, the transverse component of the polarization $\bar{P}_{T}^{\mu}\left(E_{\bar{\nu}_{\mu}}\right)$ is sensitive to the numerical value of $g_{2}^{I}(0)$.

As discussed in the introduction, the hyperons $\left(\Lambda, \Sigma^{0}\right.$, $\Sigma^{-}$) produced in the reactions given in Eq. (1) and (2) are the most appropriate candidates for doing the polarization measurements as they are self analyzer of their polarization through the measurement of the asymmetry of the angular distribution of the pions as their decay product. Out of the $\Lambda, \Sigma^{0}$ and $\Sigma^{-}$hyperons, $\Lambda$ production cross sections are the largest. Therefore, we have studied the $Q^{2}$ dependence of the cross section $\left(d \sigma / d Q^{2}\right)$ and the polarization components $P_{L, P, T}\left(Q^{2}\right)$ for various 

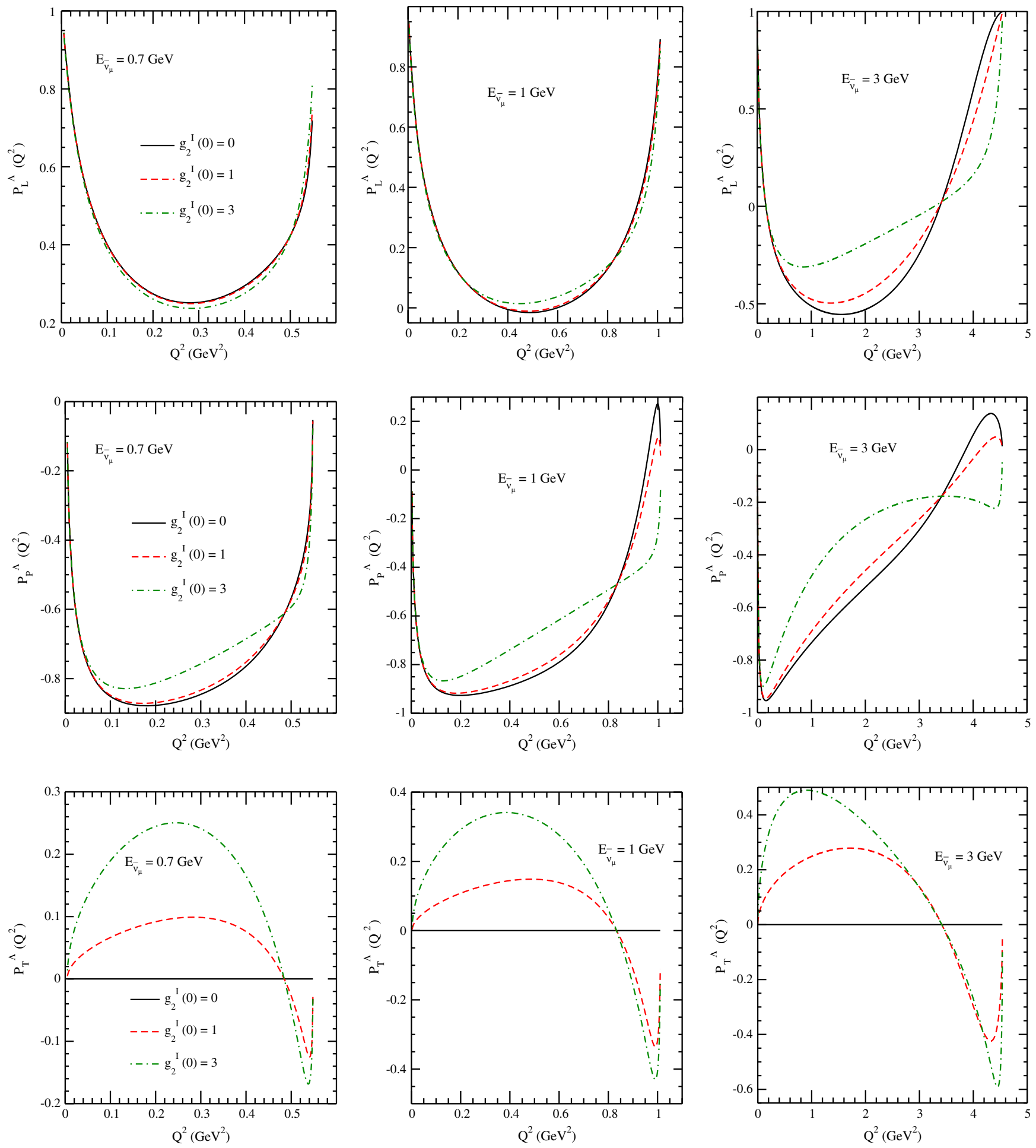

FIG. 17. $P_{L}^{\Lambda}\left(Q^{2}\right)$ vs. $Q^{2}$ (upper panel), $P_{P}^{\Lambda}\left(Q^{2}\right)$ vs. $Q^{2}$ (middle panel) and $P_{T}^{\Lambda}\left(Q^{2}\right)$ vs. $Q^{2}$ (lower panel) for the process $\bar{\nu}_{\mu}+p \rightarrow$ $\mu^{+}+\Lambda$ at the different values of the incoming antineutrino energy, $E_{\bar{\nu}_{\mu}}=0.7 \mathrm{GeV}$ (left panel), $1 \mathrm{GeV}$ (middle panel) and $3 \mathrm{GeV}$ (right panel). Lines and points have the same meaning as in Fig. 4.

antineutrino energies and present the numerical results in the following.

In Fig. 15, we have presented the results for the $d \sigma / d Q^{2}$ for the process $\bar{\nu}_{\mu}+p \rightarrow \mu^{+}+\Lambda$ at the different values of the antineutrino energies, viz. $E_{\bar{\nu}_{\mu}}=0.7,1$ and $3 \mathrm{GeV}$ by varying $\left|g_{2}^{R}(0)\right|$ as well as $g_{2}^{I}(0)$ in the range $0-3$. We find that the $d \sigma / d Q^{2}$ is not very sensitive to the presence of SCC. 
For the reaction $\bar{\nu}_{\mu}+p \rightarrow \mu^{+}+\Lambda$, we have presented the results for $P_{L}\left(Q^{2}\right)$ and $P_{P}\left(Q^{2}\right)$ as a function of $Q^{2}$ in Fig. 16. The results are presented for the polarization components using $g_{2}^{R}(0)=0, \pm 1$ and \pm 3 at the different values of $E_{\bar{\nu}_{\mu}}=0.7,1$ and $3 \mathrm{GeV}$. We find that $P_{L}\left(Q^{2}\right)$ shows large variations as we change $\left|g_{2}^{R}(0)\right|$ from 0 to 3 at low antineutrino energies, $E_{\bar{\nu}_{\mu}}$ (say $0.7 \mathrm{GeV}$ ) in comparison to the higher energies (say $3 \mathrm{GeV}$ ). For example, in the peak region of $Q^{2}$, the difference is $80 \%$ at $E_{\nu_{\mu}}=0.7 \mathrm{GeV}$ and it is $50 \%$ at $E_{\nu_{\mu}}=3 \mathrm{GeV}$ as $\left|g_{2}^{R}(0)\right|$ is changed from 0 to 3. In the case of $P_{P}\left(Q^{2}\right)$ also, the $Q^{2}$ dependence is quite strong and similar to $P_{L}\left(Q^{2}\right)$.

In Fig. 17, the results are presented for $P_{L}\left(Q^{2}\right), P_{P}\left(Q^{2}\right)$ and $P_{T}\left(Q^{2}\right)$ as a function of $Q^{2}$ using $g_{2}^{I}(0)=0,1$ and 3 at the different values of $E_{\bar{\nu}_{\mu}}=0.7,1$ and $3 \mathrm{GeV}$. We see that while $P_{L}\left(Q^{2}\right)$ is less sensitive to $g_{2}^{I}(0)$ at low antineutrino energies, the sensitivity increases at higher antineutrino energies. $P_{P}\left(Q^{2}\right)$ is sensitive to $g_{2}^{I}(0)$ in the antineutrino energy range $0.7-3 \mathrm{GeV}$ and the difference increases with the increase in antineutrino energy as $g_{2}^{I}(0)$ increases from 0 to 3 . Moreover, $P_{T}\left(Q^{2}\right)$ is sensitive to $g_{2}^{I}(0)$ at all antineutrino energies. $P_{T}\left(Q^{2}\right)$ shows $25 \%, 40 \%$ and $50 \%$ variations at $Q^{2}=0.25,0.4$ and $1 \mathrm{GeV}^{2}$ at $E_{\bar{\nu}_{\mu}}=0.7$, 1 and $3 \mathrm{GeV}$, respectively, when $g_{2}^{I}(0)$ is varied from 0 to 3 .

\section{SUMMARY AND CONCLUSIONS}

We have studied in this work the quasielastic scattering of neutrinos and antineutrinos from nucleons induced by the weak charged currents in the $\Delta S=0$ and $\Delta S=1$ sectors in the presence of the SCC with and without assuming the validity of TRI. The effect of the SCC has been studied on the total cross section $\sigma\left(E_{\nu_{\mu}\left(\bar{\nu}_{\mu}\right)}\right)$ and the average polarization components of the leptons $(l=$ muon $)$ and the baryons $(h=n, p, \Lambda$, $\left.\Sigma^{0}, \Sigma^{-}\right)$in the longitudinal, perpendicular, and transverse $\bar{P}_{L, P, T}^{l, h}\left(E_{\nu_{\mu}\left(\bar{\nu}_{\mu}\right)}\right)$ components of these particles produced in the final state. In the case of the quasielastic production of $\Lambda$ hyperons by the antineutrinos, which is the most suitable candidate for measuring the polarization observables of the hyperons, we have also studied the $Q^{2}$ dependence of the polarization components $P_{L, P, T}^{\Lambda}\left(Q^{2}\right)$ and the differential cross sections $d \sigma / d Q^{2}$.

The standard parametrizations of the electroweak form factors for the first class vector and axial vector currents in the $\Delta S=0$ sector using the hypothesis of CVC and PCAC have been used for the numerical calculations. For the form factors corresponding to the SCC, the induced scalar form factor $f_{3}^{n p}\left(Q^{2}\right)$ is assumed to be zero. The induced tensor form factor $g_{2}^{n p}\left(Q^{2}\right)$ is parametrized in the dipole form with $g_{2}^{n p}(0)$ taken to be either purely real or purely imaginary with $M_{2}=M_{A}$. A purely real (imaginary) value of $g_{2}^{n p}(0)$ implies the presence of the SCC with (without) TRI.

In the $\Delta S=1$ sector, the electroweak form factors for the nucleon-hyperon transition corresponding to the first class vector and axial vector currents $f_{1}^{N Y}\left(Q^{2}\right), f_{2}^{N Y}\left(Q^{2}\right)$ and $g_{1}^{N Y}\left(Q^{2}\right)$ for $Y=\Lambda, \Sigma^{0}, \Sigma^{-}$have been calculated, respectively, in terms of the electroweak form factors of the nucleon and the parameters defined by the analysis of SHD using SU(3) symmetry. In the case of the induced pseudoscalar form factor $g_{3}^{N Y}\left(Q^{2}\right)\left(Y=\Lambda, \Sigma^{0}, \Sigma^{-}\right)$, a generalized GT relation given by Nambu [83] has been used to relate it to $g_{1}^{N Y}\left(Q^{2}\right)$. In the case of the SCC, again we assume the induced scalar form factor $f_{3}^{N Y}\left(Q^{2}\right)=0$ $\left(Y=\Lambda, \Sigma^{0}, \Sigma^{-}\right)$and use the $\mathrm{SU}(3)$ relations to relate the induced tensor form factor $g_{2}^{N Y}\left(Q^{2}\right)$ to $g_{2}^{n p}\left(Q^{2}\right)$ which is parametrized as described in the case of $\Delta S=0$ reactions.

We summarize our results in the following

(A) Total scattering cross section:

(i) The total cross section $\sigma\left(E_{\nu_{\mu}\left(\bar{\nu}_{\mu}\right)}\right)$ due to the presence of the SCC in the axial vector sector (induced tensor term), i.e., $g_{2}^{N X}\left(Q^{2}\right) \neq$ $0\left(X=p, n, \Lambda, \Sigma^{0}, \Sigma^{-}\right)$increases more with energy $E_{\nu_{\mu}\left(\bar{\nu}_{\mu}\right)}$ as compared to $g_{2}^{N X}\left(Q^{2}\right)=0$. The additional increase is significant only if $g_{2}^{N X}(0) \geq 1$. The presence of SCC with or without TRI would lead to a smaller value of axial dipole mass $M_{A}$ by about $10 \%$ in the case of $g_{2}^{R}(0)=2$ for the neutrino induced processes and $g_{2}^{R}(0)=1$ for the antineutrino induced processes. This will be in addition to the reduction due to the inclusion of meson exchange current (MEC) and nucleon correlations [87-93].

(ii) For a given value of $g_{2}^{n p}(0)$ taken to be purely real in the case of SCC with TRI, the increase in $\sigma\left(E_{\nu_{\mu}\left(\bar{\nu}_{\mu}\right)}\right)$ is almost the same for the positive as well as the negative values of $g_{2}^{n p}(0)$ as the cross section depends mainly on $\left|g_{2}^{n_{p}}\left(Q^{2}\right)\right|^{2}$ and very mildly on the interference between the first and second class currents involving $g_{2}^{R}(0)$.

(iii) In the case of $\Delta S=1$ sector, for the case of SCC with TRI, i.e., for the real values of $g_{2}^{N Y}(0)\left(Y=\Lambda, \Sigma^{0}, \Sigma^{-}\right)$, the additional increase in $\sigma\left(E_{\bar{\nu}_{\mu}}\right)$ is larger for $g_{2}^{N X}(0)<0$ than for $g_{2}^{N X}(0)>0$.

(iv) For a given value of $g_{2}^{N X}(0)$ taken to be purely imaginary in the case of SCC without TRI, the increase is the same and is independent of the sign of $g_{2}^{N X}(0)$ as its contribution is proportional to $\left|g_{2}^{N X}\left(Q^{2}\right)\right|^{2}$. 
(B) Average polarizations:

(1) Leptons

(i) The longitudinal component of the polarization $\bar{P}_{L}^{\mu}\left(E_{\nu_{\mu}}\right)$ is almost always close to -1 for $\mu^{-}$and +1 for $\mu^{+}$in reactions $\nu_{\mu}+n \rightarrow \mu^{-}+p$ and $\bar{\nu}_{\mu}+p \rightarrow \mu^{+}+n$.

(ii) The perpendicular component of the polarization $\bar{P}_{P}^{\mu}\left(E_{\nu_{\mu}}\right)$ is not very sensitive to the value of $g_{2}^{R}(0)$ unless $g_{2}^{R}(0)$ (in the case of SCC with TRI) $\geq 1$. It shows no sensitivity to $g_{2}^{I}(0)$ in the case of SCC without TRI.

(iii) The transverse component of the polarization $\bar{P}_{T}^{\mu}\left(E_{\nu_{\mu}}\right)$ is nonzero only in the presence of SCC without TRI and is sensitive to the value of $g_{2}^{I}(0)$. It could be $1 \%(3 \%)$ corresponding to $g_{2}^{I}(0)=$ 1(3) for the neutrino induced processes, and $3 \%(9 \%)$ for the antineutrino induced processes around $E_{\nu_{\mu}}=500 \mathrm{MeV}$ and decreases with energy.

(2) Hadrons

(i) The longitudinal component of the polarization $\bar{P}_{L}^{h}\left(E_{\bar{\nu}_{\mu}}\right) ;\left(h=n, p, \Lambda, \Sigma^{0}, \Sigma^{-}\right)$is negative for the proton in neutrino scattering and its absolute values decreases with energy for the positive as well as the negative values of $g_{2}^{R}(0)$ in the case of SCC with TRI. In the case of SCC without TRI, the positive as well as the negative values of $g_{2}^{I}(0)$ gives the same results and it decreases for $g_{2}^{I}(0) \geq 0$. In the case of antineutrino scattering, the polarization of neutron and hyperons $\bar{P}_{L}^{n, \Lambda, \Sigma^{-}}\left(E_{\bar{\nu}_{\mu}}\right)$ is positive at lower energies and becomes negative at higher energies and has similar behavior to the proton polarization $\bar{P}_{L}^{p}\left(E_{\nu_{\mu}}\right)$ with respect to $g_{2}^{R}(0)$ and $g_{2}^{I}(0)$.

(ii) The perpendicular component of the polarization $\bar{P}_{P}^{h}\left(E_{\bar{\nu}_{\mu}}\right)$ is always negative for proton in the neutrino scattering and its absolute values decreases with $g_{2}^{R}(0)>0$ and $\left|g_{2}^{I}(0)\right|>0$ and increases for $g_{2}^{R}(0)<0$. In the case of antineutrino scattering, the perpendicular polarization of neutrons and hyperons $\left(\Lambda, \Sigma^{-}\right)$have similar behavior to proton polarization with respect to changes in $g_{2}^{R}(0)$ and $g_{2}^{I}(0)$.

(iii) The transverse component of the polarization $\bar{P}_{T}^{h}\left(E_{\nu_{\mu}}\right)$ of the proton in the case of neutrino scattering is nonzero only in the presence of TRV and could be $15 \%(45 \%)$ for $g_{2}^{I}(0)=1(3)$ at $E_{\nu_{\mu}}=1 \mathrm{GeV}$. In the antineutrino scattering, the polarization of neutrons and hyperons have similar behavior to the proton polarization, except that quantitatively the hyperon polarization $(\Lambda$ and $\Sigma)$ are smaller than proton polarization but the neutron polarization is comparable to the proton polarization. For $g_{2}^{I}(0)<0$, the transverse polarization $\bar{P}_{T}^{n, \Lambda, \Sigma^{-}}\left(E_{\bar{\nu}_{\mu}}\right)$ changes sign while the magnitude remains unchanged.

(C) $Q^{2}$ dependence of $d \sigma / d Q^{2}$ and $P_{L, P, T}^{l, h}\left(Q^{2}\right)$ :

(i) Numerical results for the differential cross section and the polarization observables, i.e., $d \sigma / d Q^{2}$ and $P_{L, P, T}^{\Lambda}\left(Q^{2}\right)$ for $\bar{\nu}_{\mu}+p \rightarrow \mu^{+}+\Lambda$ are presented for $E_{\bar{\nu}_{\mu}}=0.7 \mathrm{GeV}, 1 \mathrm{GeV}$ and $3 \mathrm{GeV}$ taking $M_{A}=1.026 \mathrm{GeV}$ and $M_{2}=M_{A}$.

(ii) The differential cross section $d \sigma / d Q^{2}$ for all the processes considered here is not very sensitive to the presence of the SCC with or without TRI.

(iii) All the polarization components are very sensitive to the numerical values of $g_{2}^{R}(0)$ and $g_{2}^{I}(0)$ used for evaluating the polarization components $P_{L, P, T}^{\Lambda}\left(Q^{2}\right)$ and also to the sign of $g_{2}^{R}(0)$, while in case of TRV, the positive and negative values of $g_{2}^{I}(0)$ give similar results.

(D) These calculations are performed with the aim of estimating the effect of the SCC with and without TRI in the quasielastic $\nu$ and $\bar{\nu}$ scattering from the nucleon target. However, presently almost all the antineutrino experiments in the few $\mathrm{GeV}$ energy region are using nuclear targets. In the nuclear targets, these results will be modified due to the nuclear medium effects. Therefore, in a realistic situation, these calculations are required to be done in the nuclear targets relevant to the present experiments. Our future plan is to perform such calculations with nuclear medium effects which will be reported elsewhere.

We conclude that, in the era of precision experiments with neutrino and antineutrino beams, it is possible to get information on the SCC with and without TRI in the quasielastic reactions specially in the strangeness sector.

\section{ACKNOWLEDGMENTS}

M. S. A. and S. K. S. are thankful to the Department of Science and Technology (DST), Government of India, for providing financial assistance under Grant No. EMR/2016/ 002285 . 


\section{APPENDIX A: COEFFICIENTS OF THE POLARIZATION COMPONENTS OF THE OUTGOING \\ HADRON AND $N\left(Q^{2}\right)=\mathcal{J}^{\mu \nu} \mathcal{L}_{\mu \nu}$}

The expressions $A^{h}\left(Q^{2}\right), B^{h}\left(Q^{2}\right), C^{h}\left(Q^{2}\right)$, and $N\left(Q^{2}\right)$ are expressed in terms of the Mandelstam variables and the form factors as

$$
\begin{aligned}
A^{h}\left(Q^{2}\right)= & -2\left[f_{1}^{2}\left(Q^{2}\right)\left( \pm \frac{1}{2}\left(M+M^{\prime}\right)\left(\Delta^{2}-t\right)\right) \pm \frac{f_{2}^{2}\left(Q^{2}\right)}{\left(M+M^{\prime}\right)^{2}}\left(\frac{1}{2} t\left(M+M^{\prime}\right)\left(\Delta^{2}-t\right)\right)\right. \\
& \pm g_{1}^{2}\left(Q^{2}\right)\left(\frac{1}{2} \Delta\left(\left(M+M^{\prime}\right)^{2}-t\right)\right) \pm \frac{\left|g_{2}\left(Q^{2}\right)\right|^{2}}{\left(M+M^{\prime}\right)^{2}}\left(\frac{1}{2} t \Delta\left(\left(M+M^{\prime}\right)^{2}-t\right)\right) \\
& \pm \frac{f_{1}\left(Q^{2}\right) f_{2}\left(Q^{2}\right)}{\left(M+M^{\prime}\right)}\left(\frac{1}{2}\left(4 M M^{\prime} t+t^{2}-\Delta^{2}\left(M+M^{\prime}\right)^{2}\right)\right)+f_{1}\left(Q^{2}\right) g_{1}\left(Q^{2}\right)\left(-M^{\prime}\left(M^{2}+M^{\prime 2}-2 s-t\right)\right) \\
& +\frac{\operatorname{Re}\left[f_{1}\left(Q^{2}\right) g_{2}\left(Q^{2}\right)\right]}{\left(M+M^{\prime}\right)}\left(\frac{1}{2}\left(\left(M^{2}+M^{\prime 2}-2 s-t\right)\left(-t-2 M^{\prime} \Delta+\Delta^{2}\right)+m_{\mu}^{2}\left(\Delta^{2}-t\right)\right)\right) \\
& +\frac{f_{1}\left(Q^{2}\right) g_{3}\left(Q^{2}\right)}{\left(M+M^{\prime}\right)}\left(-m_{\mu}^{2}\left(\Delta^{2}-t\right)\right) \\
& +\frac{f_{2}\left(Q^{2}\right) g_{1}\left(Q^{2}\right)}{\left(M+M^{\prime}\right)}\left(\frac{1}{2}\left(\left(M^{2}-M^{\prime 2}-t\right)\left(M^{2}+M^{\prime 2}-2 s-t\right)+m_{\mu}^{2}\left(\left(M+M^{\prime}\right)^{2}-t\right)\right)\right) \\
& +\frac{\operatorname{Re}\left[f_{2}\left(Q^{2}\right) g_{2}\left(Q^{2}\right)\right]}{\left(M+M^{\prime}\right)^{2}}\left(\frac { 1 } { 2 } \left(\left(M+M^{\prime}\right)\left(\Delta^{2}-t\right)\left(m_{\mu}^{2}+M^{2}+M^{\prime 2}-2 s-t\right)\right.\right. \\
& \left.\left.+\Delta\left(m_{\mu}^{2}\left(\left(M+M^{\prime}\right)^{2}-t\right)+\left(M^{2}-M^{\prime 2}-t\right)\left(M^{2}+M^{\prime 2}-2 s-t\right)\right)\right)\right) \\
& +\frac{f_{2}\left(Q^{2}\right) g_{3}\left(Q^{2}\right)}{\left(M+M^{\prime}\right)^{2}}\left(-m_{\mu}^{2}\left(M+M^{\prime}\right)\left(\Delta^{2}-t\right)\right) \\
& \left. \pm \frac{\operatorname{Re}\left[g_{1}\left(Q^{2}\right) g_{2}\left(Q^{2}\right)\right]}{\left(M+M^{\prime}\right)}\left(\frac{1}{2}\left(\left(M+M^{\prime}\right)^{2}-t\right)\left(-\Delta^{2}-t\right)\right)\right]
\end{aligned}
$$

where $(+)$ - sign represents the (anti)neutrino induced scattering and the Mandelstam variables are defined as

$$
\begin{gathered}
s=M^{2}+2 M E, \\
t=M^{2}+M^{\prime 2}-2 M E^{\prime},
\end{gathered}
$$

with

$$
\Delta=M^{\prime}-M
$$

$$
\begin{aligned}
B^{h}\left(Q^{2}\right)= & \frac{2}{M^{\prime}}\left[f_{1}^{2}\left(Q^{2}\right)\left( \pm \frac{1}{4}\left(t\left(\Delta^{2}-2 s\right)-t^{2}-2 M^{\prime} \Delta\left(M^{2}-s\right)+m_{\mu}^{2}\left(M^{2}+2 M M^{\prime}-M^{\prime 2}+t\right)\right)\right)\right. \\
& \pm \frac{f_{2}^{2}\left(Q^{2}\right)}{\left(M+M^{\prime}\right)^{2}}\left(\frac { 1 } { 4 } \left(t\left(M+M^{\prime}\right)\left(M^{3}+M^{2} M^{\prime}-M\left(M^{\prime 2}+2 s+t\right)+M^{\prime 3}-M^{\prime} t\right)\right.\right. \\
& \left.\left.+m_{\mu}^{2}\left(M^{4}+t\left(M+M^{\prime}\right)^{2}-M^{\prime 4}\right)\right)\right) \\
& \pm g_{1}^{2}\left(Q^{2}\right)\left(\frac{1}{4}\left(\left(-2 M^{\prime}\left(M+M^{\prime}\right)\left(M^{2}-s\right)+t\left(\left(M+M^{\prime}\right)^{2}-2 s\right)-t^{2}+m_{\mu}^{2}\left(M^{2}-2 M M^{\prime}-M^{\prime 2}+t\right)\right)\right)\right. \\
& \pm \frac{\left|g_{2}\left(Q^{2}\right)\right|^{2}}{\left(M+M^{\prime}\right)^{2}}\left(\frac { 1 } { 4 } \Delta \left(2 M^{\prime}\left(-2 m_{\mu}^{2} M^{2}-M^{4}+M^{2}\left(M^{\prime 2}+s+t\right)+s\left(t-M^{\prime 2}\right)\right)\right.\right.
\end{aligned}
$$




$$
\begin{aligned}
& \left.\left.+\Delta\left(-2 M^{\prime}\left(M+M^{\prime}\right)\left(M^{2}-s\right)+t\left(\left(M+M^{\prime}\right)^{2}-2 s\right)-t^{2}\right)+m_{\mu}^{2}\left(M^{2}-2 M M^{\prime}-M^{\prime 2}+t\right)\right)\right) \\
& \pm \frac{f_{1}\left(Q^{2}\right) f_{2}\left(Q^{2}\right)}{\left(M+M^{\prime}\right)}\left(\frac { 1 } { 2 } \left(M^{4} M^{\prime}+M^{3} t-M^{2} M^{\prime}\left(M^{\prime 2}+s\right)-M t\left(M^{\prime 2}+2 s+t\right)+M^{\prime}\left(M^{\prime 2}-t\right)(s+t)\right.\right. \\
& \left.\left.+m_{\mu}^{2}\left(M^{3}+M^{2} M^{\prime}+M\left(M^{\prime 2}+t\right)-M^{\prime 3}+M^{\prime} t\right)\right)\right) \\
& +f_{1}\left(Q^{2}\right) g_{1}\left(Q^{2}\right)\left(\frac{1}{2}\left(t\left(M^{2}+M^{\prime 2}-2 s\right)-2 s\left(s-M^{2}\right)-t^{2}-m_{\mu}^{2}\left(M^{2}+M^{\prime 2}-2 s-t\right)\right)\right) \\
& +\frac{\operatorname{Re}\left[f_{1}\left(Q^{2}\right) g_{2}\left(Q^{2}\right)\right]}{\left(M+M^{\prime}\right)}\left(-\frac{1}{2}\left(M^{4} M^{\prime}-2 M^{3} s+M^{2}\left(M^{\prime 3}-M^{\prime}(s+t)-\Delta(2 s+t)\right)+2 M s(s+t)-M^{\prime 3} s\right.\right. \\
& \left.\left.-M^{\prime 2} \Delta t+M^{\prime} s t+2 \Delta s^{2}+2 \Delta s t+\Delta t^{2}+m_{\mu}^{2}\left(M^{3}+M^{2} \Delta-M(3 s+t)+\Delta\left(M^{\prime 2}-2 s-t\right)\right)+m_{\mu}^{4} M\right)\right) \\
& +\frac{f_{1}\left(Q^{2}\right) g_{3}\left(Q^{2}\right)}{\left(M+M^{\prime}\right)}\left(m_{\mu}^{2} M\left(m_{\mu}^{2}+M^{2}-s-t\right)\right) \\
& +\frac{f_{2}\left(Q^{2}\right) g_{1}\left(Q^{2}\right)}{\left(M+M^{\prime}\right)}\left(\frac { 1 } { 2 } \left(-M^{\prime}\left(M^{2}-s\right)\left(M^{2}+M^{\prime 2}-2 s\right)+t\left(M^{3}+2 M^{2} M^{\prime}+M M^{\prime 2}+M^{\prime 3}-3 M^{\prime} s\right)\right.\right. \\
& \left.\left.-t^{2}\left(M+M^{\prime}\right)-m_{\mu}^{2}\left(M^{2} M^{\prime}+M\left(M^{\prime 2}+s\right)+M^{\prime}\left(M^{\prime 2}-2 s-t\right)\right)+m_{\mu}^{4} M\right)\right) \\
& +\frac{\operatorname{Re}\left[f_{2}\left(Q^{2}\right) g_{2}\left(Q^{2}\right)\right]}{\left(M+M^{\prime}\right)^{2}}\left(\frac { 1 } { 4 } \left(2 \left(M^{3}\left(-M^{\prime 3}+M^{\prime}(3 s+t)+\Delta t\right)+M^{2}\left(M^{\prime 4}-M^{\prime 3} \Delta-M^{\prime 2}(3 s+t)\right.\right.\right.\right. \\
& \left.+M^{\prime} \Delta(3 s+2 t)+2 s t\right)+M M^{\prime} s\left(M^{\prime 2}-2 s-3 t\right)+s\left(M^{\prime 2}-t\right)\left(2(s+t)-M^{\prime 2}\right)+M \Delta t\left(M^{\prime 2}-t\right) \\
& \left.+M^{\prime} \Delta(s+t)\left(M^{\prime 2}-2 s-t\right)\right) \\
& +m_{\mu}^{2}\left(-2 M^{4}-2 M^{3} M^{\prime}+M^{2}\left(-2 M^{\prime} \Delta+2 s+t\right)-2 M \Delta\left(M^{\prime 2}+s\right)+2 M M^{\prime}(3 s+t)\right. \\
& \left.\left.\left.+2 M^{\prime} \Delta\left(-M^{\prime 2}+2 s+t\right)-\left(M^{\prime 2}-t\right)(4 s+t)\right)-m_{\mu}^{4}\left(3 M^{2}-M^{\prime 2}+t\right)\right)\right) \\
& +\frac{f_{2}\left(Q^{2}\right) g_{3}\left(Q^{2}\right)}{\left(M+M^{\prime}\right)^{2}}\left(\frac{1}{2} m_{\mu}^{2}\left(m_{\mu}^{2}\left(M^{2}+2 M M^{\prime}-M^{\prime 2}+t\right)-2 M^{\prime} \Delta\left(M^{2}-s\right)+t\left(\Delta^{2}-2 s\right)-t^{2}\right)\right) \\
& \pm \frac{\operatorname{Re}\left[g_{1}\left(Q^{2}\right) g_{2}\left(Q^{2}\right)\right]}{\left(M+M^{\prime}\right)}\left(\frac { 1 } { 2 } \left(M^{\prime}\left(-2 m_{\mu}^{2} M^{2}-M^{4}+M^{2}\left(M^{\prime 2}+s+t\right)+s\left(t-M^{\prime 2}\right)\right)\right.\right. \\
& \left.\left.\left.+\Delta\left(m_{\mu}^{2}\left(M^{2}-2 M M^{\prime}-M^{\prime 2}+t\right)-2 M^{\prime}\left(M+M^{\prime}\right)\left(M^{2}-s\right)+t\left(\left(M+M^{\prime}\right)^{2}-2 s\right)-t^{2}\right)\right)\right)\right]
\end{aligned}
$$

$$
\begin{aligned}
C^{h}\left(Q^{2}\right)= & 2\left[ \pm \frac{\operatorname{Im}\left[f_{1}\left(Q^{2}\right) g_{2}\left(Q^{2}\right)\right]}{\left(M+M^{\prime}\right)}\left(-t+2 M \Delta+\Delta^{2}\right) \pm \frac{\operatorname{Im}\left[f_{2}\left(Q^{2}\right) g_{2}\left(Q^{2}\right)\right]}{\left(M+M^{\prime}\right)}\left(-t+\frac{\Delta\left(M^{2}-M^{\prime 2}+t\right)}{M+M^{\prime}}+\Delta^{2}\right)\right. \\
& \left.+\frac{\operatorname{Im}\left[g_{1}\left(Q^{2}\right) g_{2}\left(Q^{2}\right)\right]}{\left(M+M^{\prime}\right)}\left(M^{2}+M^{\prime 2}-2 s-t+m_{\mu}^{2}\right)+\frac{\operatorname{Im}\left[g_{3}\left(Q^{2}\right) g_{2}\left(Q^{2}\right)\right]}{\left(M+M^{\prime}\right)^{2}}\left(2 m_{\mu}^{2} \Delta\right)\right]
\end{aligned}
$$




$$
\begin{aligned}
& N\left(Q^{2}\right)=f_{1}^{2}\left(Q^{2}\right)\left(\frac{1}{2}\left(2\left(M^{2}-s\right)\left(M^{\prime 2}-s\right)-t\left(\Delta^{2}-2 s\right)+t^{2}+m_{\mu}^{2}\left(\Delta^{2}-2 s-t\right)\right)\right) \\
& +\frac{f_{2}^{2}\left(Q^{2}\right)}{\left(M+M^{\prime}\right)^{2}}\left(\frac { 1 } { 4 } \left(-2 t\left(M^{4}-2 s\left(M^{2}+M^{\prime 2}\right)+M^{\prime 4}+2 s^{2}\right)+2 t^{2}\left(\left(M+M^{\prime}\right)^{2}-2 s\right)\right.\right. \\
& +m_{\mu}^{2}\left(2 \Delta\left(M+M^{\prime}\right)\left(M^{2}+M^{\prime 2}-2 s\right)+t\left(\left(M-3 M^{\prime}\right)\left(M+M^{\prime}\right)+4 s\right)+t^{2}\right) \\
& \left.\left.+m_{\mu}^{4}\left(-\left(\left(3 M-M^{\prime}\right)\left(M+M^{\prime}\right)+t\right)\right)\right)\right) \\
& +g_{1}^{2}\left(Q^{2}\right)\left(\frac{1}{2}\left(2\left(M^{2}-s\right)\left(M^{\prime 2}-s\right)-t\left(\left(M+M^{\prime}\right)^{2}-2 s\right)+t^{2}+m_{\mu}^{2}\left(\left(M+M^{\prime}\right)^{2}-2 s-t\right)\right)\right) \\
& +\frac{\left|g_{2}\left(Q^{2}\right)\right|^{2}}{\left(M+M^{\prime}\right)^{2}}\left(\frac { 1 } { 4 } \left(4\left(\Delta^{2}-t\right)\left(\left(M^{2}-s\right)\left(M^{\prime 2}-s\right)+s t\right)+m_{\mu}^{2}\left(4 \Delta\left(M^{3}+M^{2} M^{\prime}-M(3 s+t)+M^{\prime} s\right)\right.\right.\right. \\
& \left.+2 \Delta^{2}\left(\left(M+M^{\prime}\right)^{2}-2 s-t\right)-(4 s+t)\left(\Delta^{2}-t\right)\right)+2 \Delta^{2}\left(-2\left(M^{2}-s\right)\left(M^{\prime 2}-s\right)-t\left(\left(M+M^{\prime}\right)^{2}+2 s\right)+t^{2}\right) \\
& \left.\left.+m_{\mu}^{4}\left(\Delta^{2}+4 M \Delta-t\right)\right)\right) \\
& +\frac{g_{3}^{2}\left(Q^{2}\right)}{\left(M+M^{\prime}\right)^{2}}\left(m_{\mu}^{2}\left(m_{\mu}^{2}-t\right)\left(\Delta^{2}-t\right)\right) \\
& +\frac{f_{1}\left(Q^{2}\right) f_{2}\left(Q^{2}\right)}{\left(M+M^{\prime}\right)}\left(-\left(t\left(M+M^{\prime}\right)\left(\Delta^{2}-t\right)+m_{\mu}^{2}\left(-\Delta\left(M^{\prime 2}-s\right)+M^{\prime} t\right)+m_{\mu}^{4} M\right)\right) \\
& \pm f_{1}\left(Q^{2}\right) g_{1}\left(Q^{2}\right)\left(-\left(t\left(M^{2}+M^{\prime 2}-2 s-t\right)+m_{\mu}^{2}\left(M^{2}-M^{\prime 2}+t\right)\right)\right) \\
& \pm \frac{\operatorname{Re}\left[f_{1}\left(Q^{2}\right) g_{2}\left(Q^{2}\right)\right]}{\left(M+M^{\prime}\right)}\left(-\Delta\left(t\left(M^{2}+M^{\prime 2}-2 s-t\right)+m_{\mu}^{2}\left(M^{2}-M^{\prime 2}+t\right)\right)\right) \\
& \pm \frac{f_{2}\left(Q^{2}\right) g_{1}\left(Q^{2}\right)}{\left(M+M^{\prime}\right)}\left(-\left(M+M^{\prime}\right)\left(t\left(M^{2}+M^{\prime 2}-2 s-t\right)+m_{\mu}^{2}\left(M^{2}-M^{\prime 2}+t\right)\right)\right) \\
& \pm \frac{\operatorname{Re}\left[f_{2}\left(Q^{2}\right) g_{2}\left(Q^{2}\right)\right]}{\left(M+M^{\prime}\right)^{2}}\left(\Delta\left(-\left(M+M^{\prime}\right)\right)\left(t\left(M^{2}+M^{\prime 2}-2 s-t\right)+m_{\mu}^{2}\left(M^{2}-M^{\prime 2}+t\right)\right)\right) \\
& +\frac{\operatorname{Re}\left[g_{1}\left(Q^{2}\right) g_{2}\left(Q^{2}\right)\right]}{\left(M+M^{\prime}\right)}\left(\left(\Delta\left(-t\left(M+M^{\prime}\right)^{2}+t^{2}\right)+m_{\mu}^{2}\left(M^{3}+M^{2} M^{\prime}+\Delta\left(\left(M+M^{\prime}\right)^{2}-2 s-t\right)\right.\right.\right. \\
& \left.\left.\left.-3 M s-M t+M^{\prime} s\right)+m_{\mu}^{4} M\right)\right) \\
& +\frac{g_{1}\left(Q^{2}\right) g_{3}\left(Q^{2}\right)}{\left(M+M^{\prime}\right)}\left(-2 m_{\mu}^{2}\left(m_{\mu}^{2} M+M^{3}-M^{2} M^{\prime}-M(s+t)+M^{\prime} s\right)\right) \\
& +\frac{\operatorname{Re}\left[g_{2}\left(Q^{2}\right) g_{3}\left(Q^{2}\right)\right]}{\left(M+M^{\prime}\right)^{2}}\left(m _ { \mu } ^ { 2 } \left(-2 \Delta\left(m_{\mu}^{2} M+M^{3}-M^{2} M^{\prime}-M(s+t)+M^{\prime} s\right)\right.\right. \\
& \left.\left.-\left(\Delta^{2}-t\right)\left(m_{\mu}^{2}+2 M^{2}-2 s-t\right)\right)\right) \text {. }
\end{aligned}
$$

\section{APPENDIX B: COEFFICIENTS OF THE POLARIZATION COMPONENTS OF THE OUTGOING LEPTON}

The expressions $A^{l}\left(Q^{2}\right), B^{l}\left(Q^{2}\right)$ and $C^{l}\left(Q^{2}\right)$ are expressed in terms of the Mandelstam variables and the form factors as

$$
\begin{aligned}
A^{l}\left(Q^{2}\right)= & 2\left[f_{1}^{2}\left(Q^{2}\right)\left(\frac{1}{2} m_{\mu}\left(M^{2}+2 M M^{\prime}-s\right)\right)\right. \\
& +\frac{f_{2}^{2}\left(Q^{2}\right)}{\left(M+M^{\prime}\right)^{2}}\left(\frac{1}{4} m_{\mu}\left(-m_{\mu}^{2}\left(\left(3 M-M^{\prime}\right)\left(M+M^{\prime}\right)+t\right)-2 M^{4}+2 M^{2}\left(M^{\prime 2}+s\right)+4 M M^{\prime} t-2\left(M^{\prime 2}-t\right)(s+t)\right)\right) \\
& +g_{1}^{2}\left(Q^{2}\right)\left(\frac{1}{2} m_{\mu}\left(M^{2}-2 M M^{\prime}-s\right)\right)
\end{aligned}
$$




$$
\begin{aligned}
& +\frac{\left|g_{2}\left(Q^{2}\right)\right|^{2}}{\left(M+M^{\prime}\right)^{2}}\left(\frac { 1 } { 4 } m _ { \mu } \left(\left(\Delta^{2}-t\right)\left(m_{\mu}^{2}+4 M^{2}+2 M^{\prime 2}-2 s-2 t\right)+2 \Delta\left(2 m_{\mu}^{2} M-4 M^{2} \Delta\right.\right.\right. \\
& \left.\left.\left.+3 M\left(M^{\prime 2}-s-t\right)+M^{\prime}\left(-M^{\prime 2}+s+t\right)\right)+2 \Delta^{2}\left(M^{2}-2 M M^{\prime}-s\right)\right)\right) \\
& +\frac{g_{3}^{2}\left(Q^{2}\right)}{\left(M+M^{\prime}\right)^{2}}\left(m_{\mu}^{3}\left(\Delta^{2}-t\right)\right) \\
& +\frac{f_{1}\left(Q^{2}\right) f_{2}\left(Q^{2}\right)}{\left(M+M^{\prime}\right)}\left(-\frac{1}{2} m_{\mu}\left(2 m_{\mu}^{2} M-\Delta\left(2 M^{2}-M^{\prime 2}-s\right)-t\left(3 M+M^{\prime}\right)\right)\right) \\
& +f_{1}\left(Q^{2}\right) g_{1}\left(Q^{2}\right)\left(-m_{\mu}\left(M^{2}-s\right)\right)+\frac{\operatorname{Re}\left[f_{1}\left(Q^{2}\right) g_{2}\left(Q^{2}\right)\right]}{\left(M+M^{\prime}\right)}\left(-m_{\mu} \Delta\left(M^{2}-s\right)\right) \\
& +\frac{f_{2}\left(Q^{2}\right) g_{1}\left(Q^{2}\right)}{\left(M+M^{\prime}\right)}\left(-m_{\mu}\left(M+M^{\prime}\right)\left(M^{2}-s\right)\right)+\frac{\operatorname{Re}\left[f_{2}\left(Q^{2}\right) g_{2}\left(Q^{2}\right)\right]}{\left(M+M^{\prime}\right)^{2}}\left(-m_{\mu} \Delta\left(M+M^{\prime}\right)\left(M^{2}-s\right)\right) \\
& +\frac{\operatorname{Re}\left[g_{1}\left(Q^{2}\right) g_{2}\left(Q^{2}\right)\right]}{\left(M+M^{\prime}\right)}\left(\frac { 1 } { 2 } m _ { \mu } \left(2 m_{\mu}^{2} M+4 M^{3}+2 M^{2}\left(\Delta-2 M^{\prime}\right)+M M^{\prime}\left(3 M^{\prime}-4 \Delta\right)\right.\right. \\
& \left.\left.-3 M(s+t)-M^{\prime 3}+M^{\prime}(s+t)-2 \Delta s\right)\right) \\
& +\frac{g_{1}\left(Q^{2}\right) g_{3}\left(Q^{2}\right)}{\left(M+M^{\prime}\right)}\left(-\left(2 m_{\mu}^{3} M-m_{\mu} \Delta\left(M^{\prime 2}-s-t\right)\right)\right) \\
& \left.+\frac{\operatorname{Re}\left[g_{3}\left(Q^{2}\right) g_{2}\left(Q^{2}\right)\right]}{\left(M+M^{\prime}\right)^{2}}\left(-m_{\mu}\left(m_{\mu}^{2}\left(\Delta^{2}+2 M \Delta-t\right)-t\left(M^{\prime 2}-s-t\right)\right)\right)\right] \\
& B^{l}\left(Q^{2}\right)=\frac{2}{M^{\prime}}\left[f_{1}^{2}\left(Q^{2}\right)\left(\frac{1}{4 m_{\mu}} M^{\prime}\left(m_{\mu}^{2}\left(-\left(M^{2}+2 M M^{\prime}-M^{\prime 2}+t\right)\right)+2\left(M^{2}-s\right)\left(M^{\prime 2}-s\right)-t \Delta^{2}+2 s t+t^{2}\right)\right)\right. \\
& +\frac{f_{2}^{2}\left(Q^{2}\right)}{\left(M+M^{\prime}\right)^{2}}\left(\frac { 1 } { 8 m _ { \mu } } M ^ { \prime } \left(m_{\mu}^{4}\left(\left(3 M-M^{\prime}\right)\left(M+M^{\prime}\right)+t\right)+m_{\mu}^{2}\left(\Delta^{2}-t\right)\left(2\left(M+M^{\prime}\right)^{2}+t\right)\right.\right. \\
& \left.\left.-2 t\left(M^{4}-2 s\left(M^{2}+M^{\prime 2}\right)+M^{\prime 4}+2 s^{2}\right)+2 t^{2}\left(\left(M+M^{\prime}\right)^{2}-2 s\right)\right)\right) \\
& +g_{1}^{2}\left(Q^{2}\right)\left(\frac{1}{4 m_{\mu}} M^{\prime}\left(m_{\mu}^{2}\left(-\left(M^{2}-2 M M^{\prime}-M^{\prime 2}+t\right)\right)+2\left(M^{2}-s\right)\left(M^{\prime 2}-s\right)-t\left(M+M^{\prime}\right)^{2}+2 s t+t^{2}\right)\right) \\
& +\frac{\left|g_{2}\left(Q^{2}\right)\right|^{2}}{\left(M+M^{\prime}\right)^{2}}\left(\frac { 1 } { 8 m _ { \mu } } M ^ { \prime } \left(m_{\mu}^{4}\left(-\left(\Delta^{2}+4 M \Delta-t\right)\right)+m_{\mu}^{2}\left(-2 \Delta^{2}\left(M^{2}-2 M M^{\prime}-M^{\prime 2}+t\right)\right.\right.\right. \\
& \left.-\left(4 M^{2}-t\right)\left(\Delta^{2}-t\right)+4 M \Delta(2 M \Delta+t)\right)+2 \Delta^{2}\left(2\left(M^{2}-s\right)\left(M^{\prime 2}-s\right)-t\left(M+M^{\prime}\right)^{2}+2 s t+t^{2}\right) \\
& \left.\left.-8 \Delta^{2}\left(\left(M^{2}-s\right)\left(M^{\prime 2}-s\right)+s t\right)+4\left(\Delta^{2}-t\right)\left(\left(M^{2}-s\right)\left(M^{\prime 2}-s\right)+s t\right)\right)\right) \\
& +\frac{g_{3}^{2}\left(Q^{2}\right)}{\left(M+M^{\prime}\right)^{2}}\left(-\frac{1}{2} m_{\mu} M^{\prime}\left(m_{\mu}^{2}-t\right)\left(\Delta^{2}-t\right)\right) \\
& +\frac{f_{1}\left(Q^{2}\right) f_{2}\left(Q^{2}\right)}{\left(M+M^{\prime}\right)}\left(\frac{1}{2 m_{\mu}} M^{\prime}\left(m_{\mu}^{2}-t\right)\left(m_{\mu}^{2} M+\left(M+M^{\prime}\right)\left(\left(M-M^{\prime}\right)^{2}-t\right)\right)\right) \\
& +f_{1}\left(Q^{2}\right) g_{1}\left(Q^{2}\right)\left(\frac{1}{2 m_{\mu}} M^{\prime}\left(m_{\mu}^{2}-t\right)\left(M^{2}+M^{\prime 2}-2 s-t\right)\right) \\
& +\frac{\operatorname{Re}\left[f_{1}\left(Q^{2}\right) g_{2}\left(Q^{2}\right)\right]}{\left(M+M^{\prime}\right)}\left(\frac{1}{2 m_{\mu}} M^{\prime} \Delta\left(m_{\mu}^{2}-t\right)\left(M^{2}+M^{\prime 2}-2 s-t\right)\right) \\
& +\frac{f_{2}\left(Q^{2}\right) g_{1}\left(Q^{2}\right)}{\left(M+M^{\prime}\right)}\left(\frac{1}{2 m_{\mu}} M^{\prime}\left(m_{\mu}^{2}-t\right)\left(M+M^{\prime}\right)\left(M^{2}+M^{\prime 2}-2 s-t\right)\right)
\end{aligned}
$$




$$
\begin{aligned}
& +\frac{\operatorname{Re}\left[f_{2}\left(Q^{2}\right) g_{2}\left(Q^{2}\right)\right]}{\left(M+M^{\prime}\right)^{2}}\left(\frac{1}{2 m_{\mu}} M^{\prime} \Delta\left(m_{\mu}^{2}-t\right)\left(M+M^{\prime}\right)\left(M^{2}+M^{\prime 2}-2 s-t\right)\right) \\
& +\frac{\operatorname{Re}\left[g_{1}\left(Q^{2}\right) g_{2}\left(Q^{2}\right)\right]}{\left(M+M^{\prime}\right)}\left(\frac { 1 } { 2 m _ { \mu } } M ^ { \prime } \left(-m_{\mu}^{4} M+m_{\mu}^{2}\left(-2 M^{3}+M^{2}\left(2 M^{\prime}-\Delta\right)+M\left(2 M^{\prime} \Delta+t\right)+\Delta\left(M^{\prime 2}-t\right)\right)\right.\right. \\
& \left.\left.+\Delta\left(2\left(M^{2}-s\right)\left(M^{\prime 2}-s\right)-t\left(\left(M+M^{\prime}\right)^{2}-2 s\right)+t^{2}\right)-2 \Delta\left(\left(M^{2}-s\right)\left(M^{\prime 2}-s\right)+s t\right)\right)\right) \\
& +\frac{g_{1}\left(Q^{2}\right) g_{3}\left(Q^{2}\right)}{\left(M+M^{\prime}\right)}\left(m_{\mu} M M^{\prime}\left(m_{\mu}^{2}-t\right)\right) \\
& \left.+\frac{\operatorname{Re}\left[g_{2}\left(Q^{2}\right) g_{3}\left(Q^{2}\right)\right]}{\left(M+M^{\prime}\right)^{2}}\left(\frac{1}{2} m_{\mu} M^{\prime}\left(m_{\mu}^{2}-t\right)\left(\Delta^{2}+2 M \Delta-t\right)\right)\right] \\
& C^{l}\left(Q^{2}\right)=2\left[\frac{\operatorname{Im}\left[g_{1}\left(Q^{2}\right) g_{2}\left(Q^{2}\right)\right]}{\left(M+M^{\prime}\right)}\left(m_{\mu}\left(M+M^{\prime}\right)\right)+\frac{\operatorname{Im}\left[g_{3}\left(Q^{2}\right) g_{2}\left(Q^{2}\right)\right]}{\left(M+M^{\prime}\right)^{2}}\left(2 m_{\mu} t\right)\right] .
\end{aligned}
$$

[1] S. Weinberg, Phys. Rev. 112, 1375 (1958).

[2] B. R. Holstein, Weak Interaction in Nuclei (Princeton University Press, Princeton, NJ, 1990).

[3] D. H. Wilkinson, Eur. Phys. J. A 7, 307 (2000).

[4] D. H. Wilkinson, Nucl. Instrum. Methods Phys. Res., Sect. A 455, 656 (2000).

[5] J. C. Hardy and I. S. Towner, Phys. Rev. C 71, 055501 (2005).

[6] K. Minamisono et al., Phys. Rev. C 84, 055501 (2011).

[7] S. Triambak et al., Phys. Rev. C 95, 035501 (2017); 95, 049901(A) (2017).

[8] L. A. Ahrens et al., Phys. Lett. B 202, 284 (1988).

[9] N. J. Baker, A. M. Cnops, P. L. Connolly, S. A. Kahn, H. G. Kirk, M. J. Murtagh, R. B. Palmer, N. P. Samios, and M. Tanaka, Phys. Rev. D 23, 2499 (1981).

[10] S. V. Belikov et al., Z. Phys. A 320, 625 (1985).

[11] N. Cabibbo, E. C. Swallow, and R. Winston, Annu. Rev. Nucl. Part. Sci. 53, 39 (2003).

[12] S. Y. Hsueh et al., Phys. Rev. D 38, 2056 (1988).

[13] R. Oehme, R. Winston, and A. Garcia, Phys. Rev. D 3, 1618 (1971).

[14] A. Alavi-Harati et al. (KTeV Collaboration), Phys. Rev. Lett. 87, 132001 (2001).

[15] A. Garcia, Phys. Rev. D 3, 2638 (1971).

[16] D. Eimerl, Phys. Rev. D 9, 2650 (1974).

[17] P. L. Pritchett and N. G. Deshpande, Phys. Rev. D 8, 2963 (1973).

[18] R. Oehme, Phys. Lett. 38B, 532 (1972).

[19] B. H. Kellett, Phys. Rev. D 10, 2269 (1974).

[20] M.-S. Chen, F. S. Henyey, and G. L. Kane, Nucl. Phys. B114, 147 (1976).

[21] B. R. Holstein and S. B. Treiman, Phys. Rev. D 13, 3059 (1976).

[22] C. A. Dominguez, Phys. Rev. D 20, 802 (1979).
[23] J. F. Donoghue and B. R. Holstein, Phys. Rev. D 25, 206 (1982).

[24] N. Barik, B. K. Dash, and M. Das, Phys. Rev. D 32, 1725 (1985).

[25] O. Lie-Svendsen and H. Hogaasen, Z. Phys. C 35, 239 (1987).

[26] H. J. Weber, Phys. Lett. B 209, 425 (1988).

[27] L. J. Carson, R. J. Oakes, and C. R. Willcox, Phys. Rev. D 37, 3197 (1988).

[28] W. Y. P. Hwang and E. M. Henley, Phys. Rev. D 38, 798 (1988).

[29] S. N. Jena and S. Panda, Pramana 37, 47 (1991).

[30] H. Shiomi, Nucl. Phys. A603, 281 (1996).

[31] E. D. Commins and P. H. Bucksbaum, Weak Interactions of Leptons and Quarks (Cambridge University Press, Cambridge, ENgland, 1983).

[32] R. E. Marshak, Riazuddin, and C. P. Ryan, Theory of Weak Interactions in Particle Physics (Wiley, New York, 1969).

[33] A. Pais, Ann. Phys. (N.Y.) 63, 361 (1971).

[34] C. H. Llewellyn Smith, Phys. Rep. 3, 261 (1972).

[35] E. M. Henley, Annu. Rev. Nucl. Sci. 19, 367 (1969).

[36] F. Cannata, R. Leonardi, and F. Strocchi, Phys. Rev. D 1, 191 (1970).

[37] A. De Rujula and E. De Rafael, Phys. Lett. 32B, 495 (1970).

[38] S. L. Adler, Nuovo Cimento 30, 1020 (1963); 32, 509(E) (1964).

[39] S. M. Berman and M. Veltman, Phys. Lett. 12, 275 (1964).

[40] A. Fujii and Y. Yamaguchi, Prog. Theor. Phys. 33, 552 (1965).

[41] A. Fujii and Y. Yamaguchi, Nuovo Cimento 43, 325 (1966).

[42] N. Cabibbo, Phys. Lett. 12, 137 (1964).

[43] S. L. Glashow, Phys. Rev. Lett. 14, 35 (1965).

[44] H. Okamura, Prog. Theor. Phys. 45, 1707 (1971).

[45] I. J. Ketley, Nuovo Cimento 38, 302 (1965). 
[46] L. Egardt, Nuovo Cimento 29, 954 (1963).

[47] M. M. Block, in Symmetries in Elementary Particle Physics, edited by A. Zichichi (Academic Press, New York, 1965).

[48] M. Block, NAL Summer Study, 1, 215 (1968).

[49] D. D. Javannic and M. M. Block, NAL Summer Study, 0014, 231 (1969).

[50] O. Erriquez et al., Nucl. Phys. B140, 123 (1978).

[51] J. Cao et al., arXiv:1704.08181.

[52] D. Brailsford (DUNE Collaboration), arXiv:1804.04979.

[53] C. Adams et al. (MicroBooNE Collaboration), J. Instrum. 13, P07006 (2018).

[54] S. Tufanli (SBND and MicroBooNE Collaborations), Proc. Sci., EPS-HEP2017 (2017) 141.

[55] A. Longhin (T2K Collaboration), EPJ Web Conf. 164, 01017 (2017).

[56] F. Jediny (NOvA Collaboration), Proc. Sci., FPCP2017 (2017) 025.

[57] C. E. Patrick et al. (MINERvA Collaboration), Phys. Rev. D 97, 052002 (2018).

[58] K. Abe et al., Prog. Theor. Exp. Phys. 2018, 063 C01 (2018).

[59] M. Antonello et al. (MicroBooNE and LAr1-ND, and ICARUS-WA104 Collaborations), arXiv:1503.01520.

[60] F. Akbar, M. Rafi Alam, M. Sajjad Athar, S. Chauhan, S. K. Singh, and F. Zaidi, Int. J. Mod. Phys. E 24, 1550079 (2015).

[61] M. Day and K. S. McFarland, Phys. Rev. D 86, 053003 (2012).

[62] S. M. Bilenky and E. Christova, J. Phys. G 40, 075004 (2013).

[63] S. M. Bilenky and E. Christova, Phys. Part. Nucl. Lett. 10, 651 (2013).

[64] F. Akbar, M. Rafi Alam, M. Sajjad Athar, and S. K. Singh, Phys. Rev. D 94, 114031 (2016).

[65] A. Fatima, M. Sajjad Athar, and S. K. Singh, Eur. Phys. J. A 54, 95 (2018).

[66] F. Akbar, M. Sajjad Athar, A. Fatima, and S. K. Singh, Eur. Phys. J. A 53, 154 (2017).

[67] K. M. Graczyk and B. E. Kowal, Phys. Rev. D 97, 013001 (2018).

[68] K. S. Kuzmin, V. V. Lyubushkin, and V. A. Naumov, Mod. Phys. Lett. A 19, 2815 (2004); [Phys. Part. Nucl. 35, S133 (2004)].

[69] K. S. Kuzmin, V. V. Lyubushkin, and V. A. Naumov, Mod. Phys. Lett. A 19, 2919 (2004).

[70] K. M. Graczyk, Nucl. Phys. B, Proc. Suppl. 139, 150 (2005).

[71] K. Hagiwara, K. Mawatari, and H. Yokoya, Nucl. Phys. B, Proc. Suppl. 139, 140 (2005).
[72] K. M. Graczyk, Nucl. Phys. A748, 313 (2005).

[73] M. Valverde, J. E. Amaro, J. Nieves, and C. Maieron, Phys. Lett. B 642, 218 (2006).

[74] V. Punjabi, C. F. Perdrisat, M. K. Jones, E. J. Brash, and C. E. Carlson, Eur. Phys. J. A 51, 79 (2015).

[75] V. Punjabi and C. F. Perdrisat, EPJ Web Conf. 66, 06019 (2014).

[76] F. E. Close, An Introduction to Quarks and Partons, (Academic Press Inc., London, 1979).

[77] P. Renton, Electroweak Interactions, (Cambridge University Press, Cambridge, 1990).

[78] R. Bradford, A. Bodek, H. Budd, and J. Arrington, Nucl. Phys. B, Proc. Suppl. 159, 127 (2006).

[79] V. Bernard, L. Elouadrhiri, and U. G. Meissner, J. Phys. G 28, R1 (2002).

[80] H. W. Fearing, P. C. McNamee, and R. J. Oakes, Nuovo Cimento A 60, 10 (1969).

[81] V. Bernard, T. R. Hemmert, and U. G. Meissner, arXiv: hep-ph/9811336.

[82] M. L. Goldberger and S. B. Treiman, Phys. Rev. 111, 354 (1958).

[83] Y. Nambu, Phys. Rev. Lett. 4, 380 (1960).

[84] S. M. Bilenky, Basics of Introduction to Feynman Diagrams and Electroweak Interactions Physics (Editions Frontières, 1994).

[85] V. Lyubushkin et al. (NOMAD Collaboration), Eur. Phys. J. C 63, 355 (2009).

[86] J. A. Formaggio and G. P. Zeller, Rev. Mod. Phys. 84, 1307 (2012).

[87] J. E. Amaro, M. B. Barbaro, J. A. Caballero, A. De Pace, T. W. Donnelly, G. D. Megias, and I. Ruiz Simo, Phys. Rev. C 95, 065502 (2017).

[88] G. D. Megias, J. E. Amaro, M. B. Barbaro, J. A. Caballero, T. W. Donnelly, and I. Ruiz Simo, Phys. Rev. D 94, 093004 (2016).

[89] A. V. Butkevich and S. V. Luchuk, Phys. Rev. C 97, 045502 (2018).

[90] M. Martini, M. Ericson, and G. Chanfray, Phys. Rev. C 84, 055502 (2011).

[91] M. Martini and M. Ericson, Phys. Rev. C 87, 065501 (2013).

[92] J. Nieves, I. Ruiz Simo, and M. J. Vicente Vacas, Phys. Lett. B 707, 72 (2012).

[93] J. Nieves, I. Ruiz Simo, and M. J. Vicente Vacas, Phys. Lett. B 721, 90 (2013). 